\title{
NLP Scholar: An Interactive Visual Explorer for Natural Language Processing Literature
}

\author{
Saif M. Mohammad \\ National Research Council Canada \\ saif.mohammadenrc-cnrc.gc.ca
}

\begin{abstract}
As part of the NLP Scholar project, we created a single unified dataset of NLP papers and their meta-information (including citation numbers), by extracting and aligning information from the ACL Anthology and Google Scholar. In this paper, we describe several interconnected interactive visualizations (dashboards) that present various aspects of the data. Clicking on an item within a visualization or entering query terms in the search boxes filters the data in all visualizations in the dashboard. This allows users to search for papers in the area of their interest, published within specific time periods, published by specified authors, etc. The interactive visualizations presented here, and the associated dataset of papers mapped to citations, have additional uses as well including understanding how the field is growing (both overall and across sub-areas), as well as quantifying the impact of different types of papers on subsequent publications.
\end{abstract}

\section{Introduction}

NLP is a broad interdisciplinary field that draws knowledge from Computer Science, Linguistics, Information Science, Psychology, Social Sciences, and more. ${ }^{1}$ Over the years, scientific publications in NLP have grown in number and diversity; we now see papers published on a vast array of research questions and applications in a growing list of venues-in journals such as CL and TACL, in large conferences such as ACL and EMNLP, as well as a number of small area-focused workshops.

The ACL Anthology (AA) is a digital repository of public domain, free to access, articles on NLP. ${ }^{2}$ It includes papers published in the family of ACL conferences as well as in other NLP conferences

\footnotetext{
${ }^{1}$ One can make a distinction between NLP and Computational Linguistics; however, for this work we will consider them to be synonymous.

${ }^{2}$ https://www.aclweb.org/anthology/
}

such as LREC and RANLP. As of June 2019, it provided access to the full text and metadata for close to $50 \mathrm{~K}$ articles published since $1965 .^{3}$ It is the largest single source of scientific literature on NLP. However, the meta-data does not include citation statistics.

Citation statistics are the most commonly used metrics of research impact. They include: number of citations, average citations, h-index, relative citation ratio, and impact factor. Note, however, that the number of citations is not always a reflection of the quality or importance of a piece of work. Furthermore, the citation process can be abused, for example, by egregious self-citations (Ioannidis et al., 2019). Nonetheless, given the immense volume of scientific literature, the relative ease with which one can track citations using services such as Google Scholar (GS), and given the lack of other easily applicable and effective metrics, citation analysis is an imperfect but useful window into research impact.

Google Scholar is a free web search engine for academic literature. ${ }^{4}$ Through it, users can access the metadata associated with an article such as the number of citations it has received. Google Scholar does not provide information on how many articles are included in its database. However, scientometric researchers estimated that it included about 389 million documents in January 2018 (Gusenbauer, 2019)—making it the world's largest source of academic information. Thus, it is not surprising that there is growing interest in the use of Google Scholar information to draw inferences about scholarly research in general (Martín-Martín et al., 2018; Mingers and Leydesdorff, 2015; Orduña-Malea et al., 2014; Khabsa and Giles, 2014; Howland, 2010) and on scholarly impact in particular (Bos

\footnotetext{
${ }^{3}$ ACL licenses its papers with a Creative Commons Attribution 4.0 International License.

${ }^{4} \mathrm{https}: / /$ scholar.google.com
} 
and Nitza, 2019; Ioannidis et al., 2019; Ravenscroft et al., 2017; Bulaitis, 2017; Yogatama et al., 2011; Priem and Hemminger, 2010).

Services such as Google Scholar and Semantic Scholar cover a wide variety of academic disciplines. Wile there are benefits to this, the lack of focus on NLP literature has some drawbacks as well: e.g, the potential for too many search results that include many irrelevant papers. For example, if one is interested in NLP papers on emotion and privacy, searching for them on Google Scholar is less efficient than searching for them on a platform dedicated to NLP papers. Further, services such as Google Scholar provide minimal interactive visualizations. NLP Scholar with its focus on AA data, is not meant to replace these tools, but act as a complementary tool for dedicated visual search of NLP literature.

ACL 2020 has a special theme asking researchers to reflect on the state of NLP. In the spirit of that theme, and as part of a broader project on analyzing NLP Literature, we extracted and aligned information from the ACL Anthology (AA) and Google Scholar to create a dataset of tens of thousands of NLP papers and their citations (Mohammad, 2020c, 2019). In separate work, we have used the data to explores questions such as: how well cited are papers of different types (journal articles, conference papers, demo papers, etc.)? how well cited are papers published in different time spans? how well cited are papers from different areas of research within NLP? etc. (Mohammad, 2020a). We also explored gender gaps in Natural Language Processing research, in terms of authorship and citations (Mohammad, 2020b). In this paper we describe how we built an interactive visual explorer for this unified data, which we refer to as $N L P$ Scholar. Some notable uses of NLP Scholar are listed below:

- Search for relevant related work in various areas within NLP.

- Identify the highly cited articles on an interactive timeline.

- Identify past papers published in a venue of interest (such as ACL or LREC).

- Identify papers from the past (say ten years back) published in a venue of interest (say ACL or LREC) that have made substantial impact through citations.
- Examine changes in number of articles and number of citations in a chosen area of interest over time.

- Identify citation impact of different types of papers-e.g., short papers, shared task papers, demo papers, etc.

Even beyond the dedicated interactive visualizer described here, the underlying data with its alignment between AA and GS has potential uses in:

- Creating a web browser extension that allows users of GS to look up the aligned AA information (the full ACL BibTeX, poster, slides, access to proceedings from the same venue, etc.).

- Similarly, in the reverse direction, allowing access from AA to the GS information on the aligned paper. This could include number of citations, lists of papers citing the paper, etc.

Perhaps most importantly, though, NLP Scholar serves as a visual record of the state of NLP literature in terms of citations. We note again though, that even though this work seeks to make citation metrics more accessible for ACL Anthology papers, citation metrics are not always accurate reflections of the quality, importance, or impact of individual papers.

All of the data and interactive visualizations associated with this work are freely available through the project homepage. ${ }^{5}$

\section{Background and Related Work}

Much of the work in visualizing scientific literature has focused on showing topics of research $(\mathrm{Wu}$ et al., 2019; Heimerl et al., 2012; Lee et al., 2005). There is also notable work on visualizing communities through citation networks (Heimerl et al., 2015; Radev et al., 2016).

Various subsets of AA have been used in the past for a number of tasks, including: to study citation patterns and intent (Radev et al., 2016; Zhu et al., 2015; Nanba et al., 2011; Mohammad et al., 2009; Teufel et al., 2006; Aya et al., 2005; Pham and Hoffmann, 2003), to generate summaries of scientific articles (Qazvinian et al., 2013), to study gender disparities in NLP (Schluter, 2018), to study subtopics within NLP (Anderson et al.,

\footnotetext{
${ }^{5}$ http://saifmohammad.com/WebPages/nlpscholar.html
} 
2012), and to create corpora of scientific articles (Mariani et al., 2018; Bird et al., 2008).

However, none of these works provide an interactive visualization for users to explore NLP literature and their citations.

\section{Data}

We now briefly describe how we extracted information from the ACL Anthology and Google Scholar. (Further details about the dataset, as well as an analysis of the volume of research in NLP over the years, are available in Mohammad (2020c).)

\subsection{ACL Anthology Data}

The ACL Anthology provides access to its data through its website and a github repository (Gildea et al., 2018). ${ }^{6}$ We extracted paper title, names of authors, year of publication, and venue of publication from the repository. ${ }^{7}$

As of June 2019, AA had $~ 50 \mathrm{~K}$ entries; however, this includes forewords, schedules, etc. that are not truly research publications. After discarding them we are left with a set of 44,895 papers.

\subsection{Google Scholar Data}

Google Scholar does not provide an API to extract information about the papers. This is likely because of its agreement with publishing companies that have scientific literature behind paywalls (MartínMartín et al., 2018). We extracted citation information from Google Scholar profiles of authors who published at least three papers in the ACL Anthology. (This is explicitly allowed by GS's robots exclusion standard. This is also how past work has studied Google Scholar (Khabsa and Giles, 2014; Orduña-Malea et al., 2014; Martín-Martín et al., 2018).) This yielded citation information for 1.1 million papers in total. We will refer to this dataset as $G S-N L P$. Note that GS-NLP includes citation counts not just for NLP papers, but also for nonNLP papers published by the authors.

GS-NLP includes 32,985 of the 44,895 papers in AA (about 74\%). We will refer to this subset of the

\footnotetext{
${ }^{6}$ https://www.aclweb.org/anthology/ https://github.com/acl-org/acl-anthology

${ }^{7}$ Multiple authors can have the same name and the same authors may use multiple variants of their names in papers. The AA volunteer team handles such ambiguities using both semi-automatic and manual approaches (fixing some instances on a case-by-case basis). Additionally, the AA repository includes a file that has canonical forms of author names. Authors can provide AA with their aliases, change-of-name information, and preferred canonical name, which is then eventually recorded in the canonical-name file.
}

ACL Anthology papers as AA'. The citation analyses presented in this paper are on $\mathrm{AA}^{\prime}$. (Future work will explore visualizations on GS-NLP.)

Entries across AA and GS are aligned by matching the paper title, year of publication, and first author last name. ${ }^{8}$

\section{Building an Interactive Visualization to Explore Scientific Literature}

We now describe how we created an interactive visualization-NLP Scholar-that allows one to visually explore the data from the ACL Anthology along with citation information from Google Scholar. We first created a relational database (involving multiple tables) that stores the AA and GS data ( $\$ 4.1)$. We then loaded the database in Tableau-an interactive data visualization software — to build the visualizations ( $\$ 4.2) .{ }^{9}$

\subsection{NLP Scholar Relational Database}

Data from AA and GS is stored in four tables (tsv files): papers, authors, title-unigrams, and titlebigrams. They contain the following information:

papers: Each row corresponds to a unique paper. The columns include: paper title, year of publication, list of authors, venue of publication, number of citations at the time of data collection (June 2019), NLP Scholar paper id, ACL paper id, and some other meta-data associated with the paper.

The NLP Scholar paper id is a concatenation of the paper title, year of publication, and first author last name. (This id was also used to align entries across AA and GS).

authors: Each row corresponds to a paper-author combination. The columns include: NLP Scholar paper id, author first name, and author last name. A paper with three authors contributes three rows to the table (all three have the same paper id, but different author names).

title-unigrams: Each row corresponds to a paper title and unigram combination. The columns include: NLP Scholar paper id and paper title unigram (a word that occurs in the title of the paper). A paper with five unique words in the title

\footnotetext{
${ }^{8}$ There were marked variations in how the same venue was described in the meta-information across AA and GS; thus, venue information was not used for alignment.

${ }^{9}$ Tableau: https://www.tableau.com

Even though there are paid versions of Tableau, the visualizations built with Tableau can be freely shared with others on the world wide web. Users do not require any special software to interact with these visualization on the web.
} 
contributes five rows to the table (all five have the same paper id, but different words).

title-bigrams: Each row corresponds to a paper title and bigram combination. The columns include: NLP Scholar paper id and paper title bigram (a two-word sequence that occurs in the title of the paper). A paper with four unique bigrams in the title contributes four rows to the table (all four have the same paper id, but different bigrams).

Once the tables are loaded in Tableau, the following pairs of tables are each joined (inner join) using the NLP Scholar paper id: ${ }^{10}$ papers-authors, papers-title-unigrams, and papers-title-bigrams.

\subsection{NLP Scholar Interactive Visualization}

We developed multiple visualizations to explore various aspects of the data. We group and connect several individual visualizations in dashboards that allow one to explore several aspects of the data together. Clicking on data attributes such as year of publication or venue of publication in one visualization, filters the data in all visualizations within a dashboard to show only the relevant data.

Figure 1 shows a screenshot of the main dashboard. At the top are the number of papers-total (A1) and by year of publication (A2). This allows one to see the growth/decline of the papers over the years.

Below it, we see the number of citations-total (B1) and by year of publication (B2). For a given year, the bar is partitioned into segments corresponding to individual papers. Each segment (paper) has a height that is proportional to the number of citations it has received and assigned a colour at random. This allows one to quickly identify high-citation papers. ${ }^{11}$

Hovering over individual papers in B2 pops open an information box showing the paper title, authors, year of publication, publication venue, and \#citations. Figure 6 in the Appendix shows a blow up of B2 along with examples of the hover information box. Similarly, hovering over other parts of the dashboard shows corresponding information. (This is especially helpful, when parts of the text

\footnotetext{
${ }^{10} \mathrm{An}$ inner join selects all rows from both participating tables whose join column values match across the two tables.

${ }^{11}$ Note that since the number of colours is smaller than the number of papers, multiple papers may have the same color however, the probability of adjacent papers receiving the same colour is small-even then, the system will provide visual clues distinguishing each segment when hovering over the area.
}

are truncated or otherwise not visible due to space constraints.)

Further below, we see lists of papers (C) and authors (D) - both are ordered by number of citations. Search boxes in the bottom right (E) allow searching for papers that have particular terms in the title or searching for papers by author name. One can also restrict the search to a span of years using the slider.

Four other dashboards are also created that have the same five elements as the main dashboard (A through E), and additionally include a six element $\mathrm{F}$ to provide a focused search facility. This sixth element is a treemap that shows the most common: venues and paper types $(\mathrm{F} 1)$, title unigrams $(\mathrm{F} 2)$, title bigrams (F3), or language mentions in the title (F4). (We only show one of the four treemaps at a time to prevent overwhelming the user.) The treemaps are shown in Figures 2 to 5, respectively.

\section{Data Explorations with NLP Scholar}

Figure 1 A1 shows that the dataset includes 44,895 papers. A2 shows that the volume of papers published was considerably lower in the early years (1965 to 1989); there was a spurt in the 1990s; and substantial numbers since the year 2000. Also, note that the number of publications is considerably higher in alternate years. This is due to certain biennial conferences. Since 1998 the largest of such conferences has been LREC (In 2018 alone LREC had over 700 main conferences papers and additional papers from its 29 workshops). COLING, another biennial conference (also occurring in the even years) has about $45 \%$ of the number of main conference papers as LREC.

$\mathrm{B} 1$ shows that $\mathrm{AA}^{\prime}$ papers have received $\sim 1.2$ million citations (as of June 2019). The timeline graph in B2 shows that, with time, not only have the number of papers grown, but also the number of high-citation papers. We see a marked jump in the 1990s over the previous decades, but the 2000s are the most notable in terms of the high number of citations. The 2010s papers will likely surpass the 2000s papers in the years to come.

The most cited papers list (C) shows influential papers from machine translation, sentiment analysis, word embeddings, syntax, and semantics.

Among the authors (D), observe that Christopher Manning has not only received the most number of citations, he has also received almost three times as many citations as the next person in the list. 

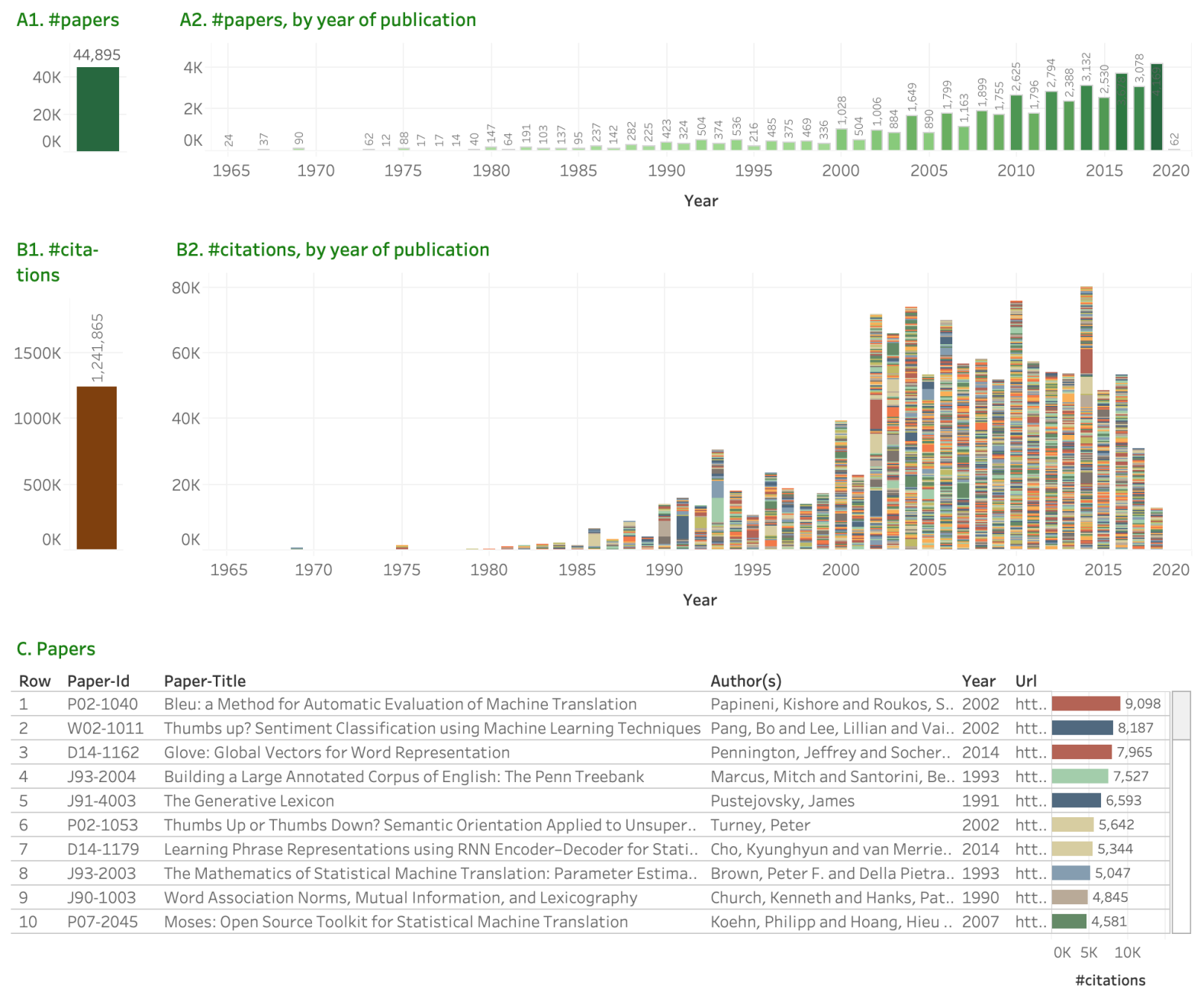

\section{Authors}

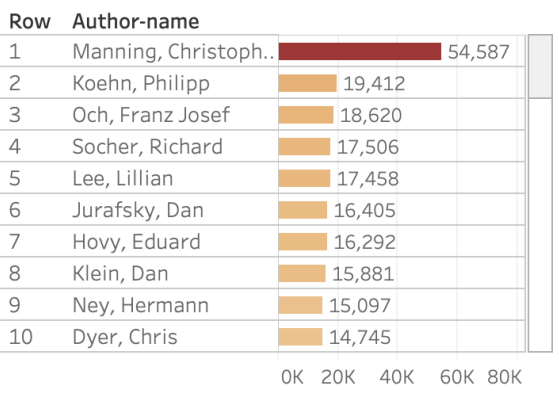

\#citations
E. Search by year of publication, title term (unigram, bigram), or author name Year of publication

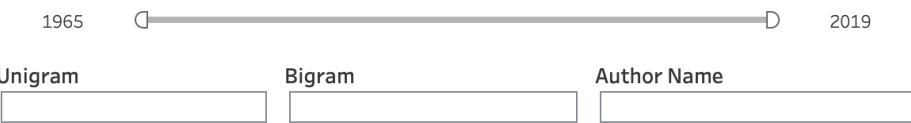

Figure 1: A screenshot of NLP Scholar's principle dashboard.

Search: NLP Scholar allows for search in a number of ways. Suppose we are interested in the topic of sentiment analysis. Then we can enter the relevant keywords in the search box: sentiment, valence, emotion, emotions, affect, etc. Then the visualizations are filtered to present details of only those papers that have at least one of these keywords in the title. (Future work will allow for search in the abstract and the whole text.)
Figure 7 in the Appendix shows the filtered result. The system identified 1,481 papers that each have at least one of the query terms in the title. They have received more than $85 \mathrm{~K}$ citations. The citations timeline (B2 in Figure 7) shows that there were just a few scattered papers in early years (1987-2000) that received a small number of citations. However, two papers in 2002 received a massive number of citations, and likely led to 
F1. Venue and Paper Type

\begin{tabular}{|c|c|c|c|c|c|c|c|c|}
\hline $\begin{array}{l}\text { *SEM } \\
192\end{array}$ & $\begin{array}{l}\mathrm{CL} \\
922\end{array}$ & $\begin{array}{l}\text { Demos } \\
981\end{array}$ & $\begin{array}{l}\text { EMNLP } \\
3,140\end{array}$ & $\begin{array}{l}\text { JEP/TALN/RECI } \\
48\end{array}$ & $\begin{array}{l}\text { NAACL } \\
1,477\end{array}$ & $\begin{array}{l}\text { ROCLING/IJCLCL } \\
918\end{array}$ & $\begin{array}{l}\text { TACL } \\
258\end{array}$ & $\begin{array}{l}\text { TINLAP } \\
84\end{array}$ \\
\hline $\begin{array}{l}\mathrm{ACL} \\
4,830\end{array}$ & $\begin{array}{l}\text { COLING } \\
3,863\end{array}$ & $\begin{array}{l}\text { Doctoral Cons. } \\
10\end{array}$ & $\begin{array}{l}\mathrm{HLT} \\
74\end{array}$ & $\begin{array}{l}\text { LREC } \\
5,763\end{array}$ & $\begin{array}{l}\text { PACLIC } \\
1,210\end{array}$ & $\begin{array}{l}\text { SEMEVAL } \\
1,347\end{array}$ & $\begin{array}{l}\text { Tutorials } \\
211\end{array}$ & \\
\hline $\begin{array}{l}\text { ANLP } \\
300\end{array}$ & $\begin{array}{l}\text { CoNLL } \\
571\end{array}$ & $\begin{array}{l}\text { EACL } \\
1,057\end{array}$ & $\begin{array}{l}\text { IJCNLP } \\
794\end{array}$ & $\begin{array}{l}\text { MUC } \\
146\end{array}$ & $\begin{array}{l}\text { RANLP } \\
501\end{array}$ & $\begin{array}{l}\text { Shared Task } \\
283\end{array}$ & $\begin{array}{l}\text { Workshops } \\
15,389\end{array}$ & \\
\hline
\end{tabular}

Figure 2: A treemap of popular NLP venues and paper types. Darker shades of green: higher volumes of papers.

F2. Title Unigrams

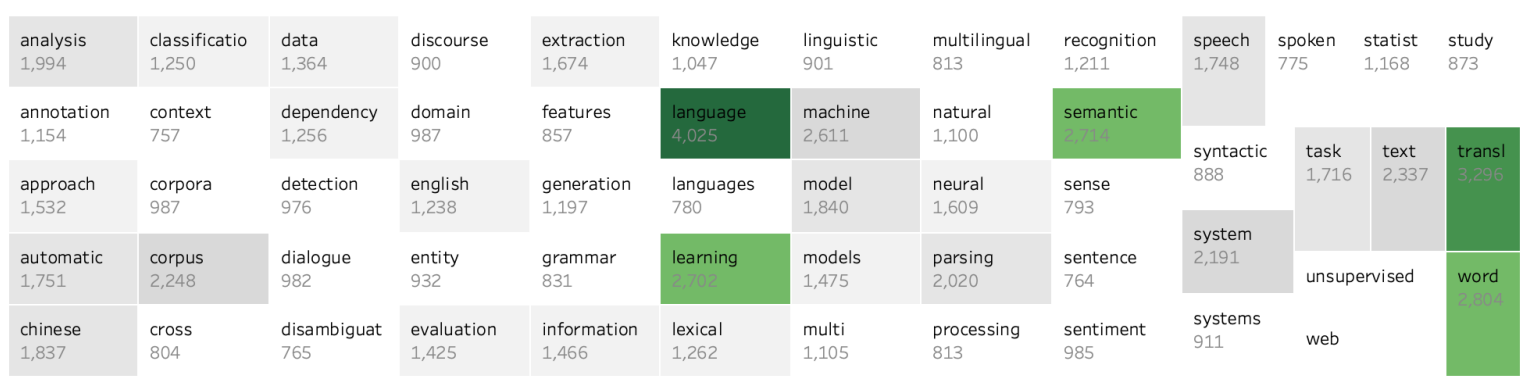

Figure 3: A treemap of the most common unigrams in paper titles. Darker shades of green: higher frequencies.

F3. Title Bigrams

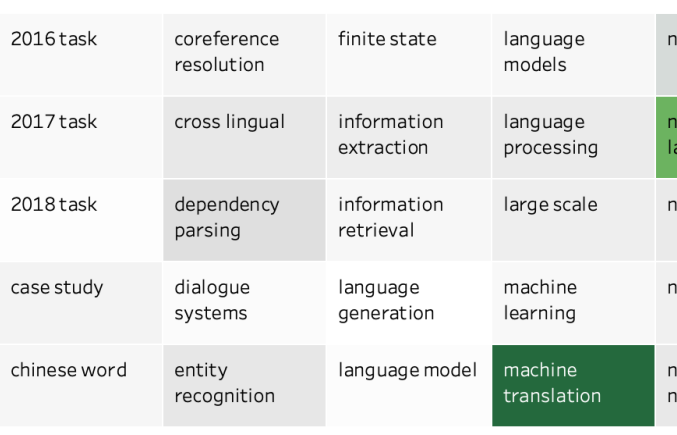

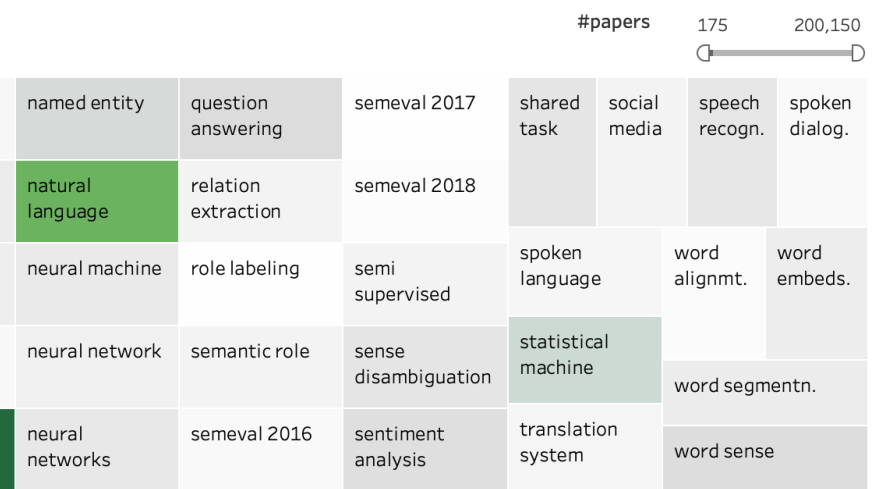

Figure 4: A treemap of the most common bigrams in paper titles. Darker shades of green: higher frequencies.

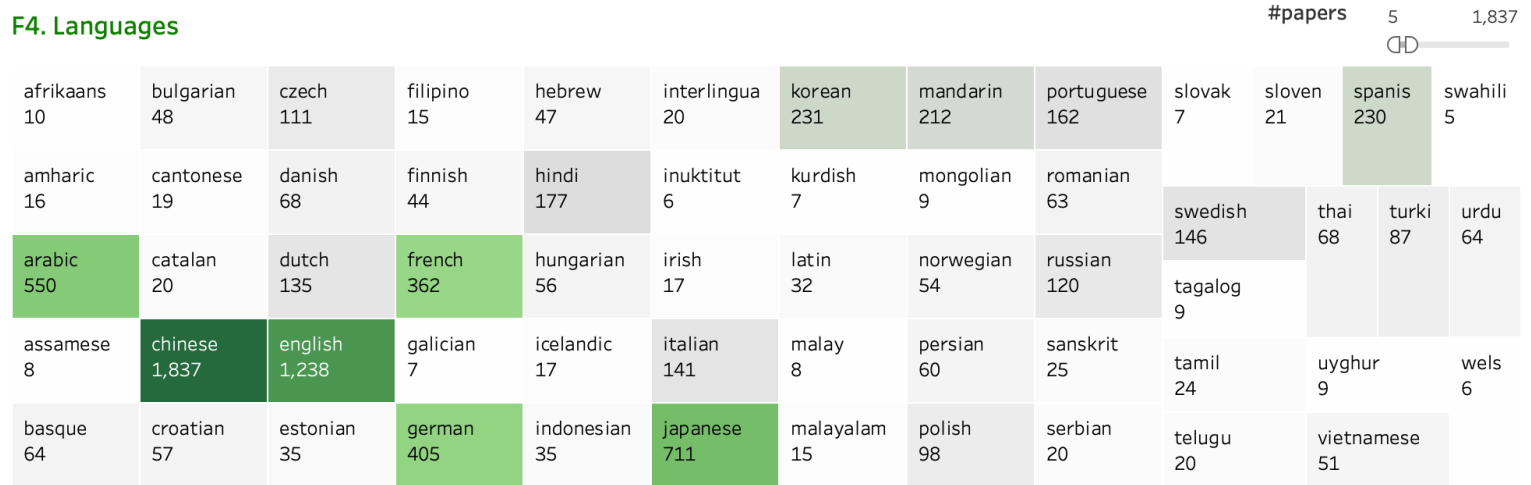

Figure 5: A treemap of the most common language terms in titles. Darker shades of green: higher frequencies. 
the substantially increased interest in the field. The number of papers has steadily increased since 2002 , with close to 250 papers in 2018 , showing that the area continues to enjoy considerable attention.

One can also fine tune the search as desired. Say we are interested not in the broad area of sentiment analysis, but specifically in the work on emotions and affect. Then they can enter only emotion- and affect-related keywords. A disadvantage of using terms for search is that some terms are ambiguous and they can pull in irrelevant articles; also if a paper is about the topic of interest but its title does not have one of the standard keywords associated with the topic, then it might be left out. That said, if one does come across a paper that has the query term but is not in the topic of interest, they can right click and exclude that paper from the visualization; and as mentioned before, future work will allow for searches in the abstract and full text as well. We are also currently working on clustering papers using the words in the articles as features. ${ }^{12}$

Below are some more examples of interactions with NLP Scholar (Figures are in the Appendix after references):

- Figure 8 shows the state of the visualization when one clicks the year 2016 in A1.

- Figures 9 and 10 show examples of author search by clicking on the authors list (D) (Christopher Manning and Lillian Lee).

- Figures 11 and 12 show the dashboard when one clicks on the Venue and Paper Type treemap (F1): ACL main conference papers and Workshop papers, respectively.

- Figures 13, 14 and 15 in the Appendix also show examples of search for the terms parsing, statistical and neural, respectively (accessed by clicking on the title unigrams treemap (F2)).

- Figures 16, 17, and 18 show the dashboard when one clicks on the Title Bigrams treemap (F3): machine translation, question answering, and word embeddings, respectively.

- Figures 19 and 20 show the dashboard when one clicks on the Languages treemap (F4): Chinese and Swahili, respectively.

\footnotetext{
${ }^{12}$ Note that clustering approaches also have limitations, such as differing results depending on the parameters used.
}

Once the system goes live, we hope to collect further usage scenarios from the users at large.

For this work, we chose not to stem the terms in the titles before applying the search. This is because in some search scenarios, it is beneficial to distinguish the different morphological forms of a word. For example, papers with emotions in the titles are more likely to be dealing with multiple emotions than papers with the term emotion. When such distinctions do not need to be made, it is easy for users to include morphological variants as additional query terms.

\section{Conclusions and Future Work}

We presented NLP Scholar-an interactive visual explorer for the ACL Anthology. Notably, the tool also has access to citation information from Google Scholar. It includes several interconnected interactive visualizations (dashboards) that allow users to quickly and efficiently search for relevant related work by clicking on items within a visualization or through search boxes. All of the data and interactive visualizations associated with this work are freely available through the project homepage. ${ }^{13}$

Future work will provide additional functionalities such as search within abstracts and whole texts, document clustering, and automatically identifying related papers. We see NLP Scholar, with its dedicated visual search capabilities for NLP papers, as a useful complementary tool to existing resources such as Google Scholar. We also note that the approach presented here is not required to be applied only to the ACL Anthology or NLP papers; it can be used to display papers from other sources too such as pre-print archives and anthologies of papers from other fields of study.

\section{Acknowledgments}

This work was possible due to the helpful discussion and encouragement from a number of awesome people including: Dan Jurafsky, Tara Small, Michael Strube, Cyril Goutte, Eric Joanis, Matt Post, Torsten Zesch, Ellen Riloff, Iryna Gurevych, Rebecca Knowles, Isar Nejadgholi, and Peter Turney. Also, a big thanks to the ACL Anthology and Google Scholar Teams for creating and maintaining wonderful resources.

\footnotetext{
${ }^{13} \mathrm{http} / / /$ saifmohammad.com/WebPages/nlpscholar.html
} 


\section{References}

Ashton Anderson, Dan McFarland, and Dan Jurafsky. 2012. Towards a computational history of the acl: 1980-2008. In Proceedings of the ACL-2012 Special Workshop on Rediscovering 50 Years of Discoveries, pages 13-21. Association for Computational Linguistics.

Selcuk Aya, Carl Lagoze, and Thorsten Joachims. 2005. Citation classification and its applications. In Knowledge Management: Nurturing Culture, Innovation, and Technology, pages 287-298. World Scientific.

Steven Bird, Robert Dale, Bonnie J Dorr, Bryan Gibson, Mark Thomas Joseph, Min-Yen Kan, Dongwon Lee, Brett Powley, Dragomir R Radev, and Yee Fan Tan. 2008. The acl anthology reference corpus: A reference dataset for bibliographic research in computational linguistics.

Arthur R Bos and Sandrine Nitza. 2019. Interdisciplinary comparison of scientific impact of publications using the citation-ratio. Data Science Journal, 18(1).

Zoe Bulaitis. 2017. Measuring impact in the humanities: Learning from accountability and economics in a contemporary history of cultural value. Palgrave Communications, 3(1):7.

Daniel Gildea, Min-Yen Kan, Nitin Madnani, Christoph Teichmann, and Martín Villalba. 2018. The ACL anthology: Current state and future directions. In Proceedings of Workshop for NLP Open Source Software (NLP-OSS), pages 23-28, Melbourne, Australia. Association for Computational Linguistics.

Michael Gusenbauer. 2019. Google scholar to overshadow them all? comparing the sizes of 12 academic search engines and bibliographic databases. Scientometrics, 118(1):177-214.

Florian Heimerl, Qi Han, Steffen Koch, and Thomas Ertl. 2015. Citerivers: Visual analytics of citation patterns. IEEE transactions on visualization and computer graphics, 22(1):190-199.

Florian Heimerl, Steffen Koch, Harald Bosch, and Thomas Ertl. 2012. Visual classifier training for text document retrieval. IEEE Transactions on Visualization and Computer Graphics, 18(12):2839-2848.

Jared L Howland. 2010. How scholarly is google scholar? a comparison to library databases.

John PA Ioannidis, Jeroen Baas, Richard Klavans, and Kevin W Boyack. 2019. A standardized citation metrics author database annotated for scientific field. PLoS biology, 17(8):e3000384.

Madian Khabsa and C Lee Giles. 2014. The number of scholarly documents on the public web. PloS one, 9(5):e93949.
Bongshin Lee, Mary Czerwinski, George Robertson, and Benjamin B Bederson. 2005. Understanding research trends in conferences using paperlens. In CHI'05 extended abstracts on Human factors in computing systems, pages 1969-1972.

Joseph Mariani, Gil Francopoulo, and Patrick Paroubek. 2018. The nlp4nlp corpus (i): 50 years of publication, collaboration and citation in speech and language processing. Frontiers in Research Metrics and Analytics, 3:36.

Alberto Martín-Martín, Enrique Orduna-Malea, Mike Thelwall, and Emilio Delgado López-Cózar. 2018. Google scholar, web of science, and scopus: A systematic comparison of citations in 252 subject categories. Journal of Informetrics, 12(4):1160-1177.

John Mingers and Loet Leydesdorff. 2015. A review of theory and practice in scientometrics. European journal of operational research, 246(1):1-19.

Saif Mohammad, Bonnie Dorr, Melissa Egan, Ahmed Hassan, Pradeep Muthukrishan, Vahed Qazvinian, Dragomir Radev, and David Zajic. 2009. Using citations to generate surveys of scientific paradigms. In Proceedings of human language technologies: The 2009 annual conference of the North American chapter of the association for computational linguistics, pages 584-592.

Saif M. Mohammad. 2019. The state of nlp literature: A diachronic analysis of the acl anthology. arXiv preprint arXiv:1911.03562.

Saif M. Mohammad. 2020a. Examining citations of natural language processing literature. In Proceedings of the 2020 Annual Conference of the Association for Computational Linguistics, Seattle, USA.

Saif M. Mohammad. 2020b. Gender gap in natural language processing research: Disparities in authorship and citations. In Proceedings of the $2020 \mathrm{An}$ nual Conference of the Association for Computational Linguistics, Seattle, USA.

Saif M. Mohammad. 2020c. Nlp scholar: A dataset for examining the state of nlp research. In Proceedings of the 12th Language Resources and Evaluation Conference (LREC-2020), Marseille, France.

Hidetsugu Nanba, Noriko Kando, and Manabu Okumura. 2011. Classification of research papers using citation links and citation types: Towards automatic review article generation. Advances in Classification Research Online, 11(1):117-134.

Enrique Orduña-Malea, Juan Manuel Ayllón, Alberto Martín-Martín, and Emilio Delgado López-Cózar. 2014. About the size of google scholar: playing the numbers. arXiv preprint arXiv:1407.6239.

Son Bao Pham and Achim Hoffmann. 2003. A new approach for scientific citation classification using cue phrases. In Australasian Joint Conference on Artificial Intelligence, pages 759-771. Springer. 
Jason Priem and Bradely H Hemminger. 2010. Scientometrics 2.0: New metrics of scholarly impact on the social web. First monday, 15(7).

Vahed Qazvinian, Dragomir R Radev, Saif M Mohammad, Bonnie Dorr, David Zajic, Michael Whidby, and Taesun Moon. 2013. Generating extractive summaries of scientific paradigms. Journal of Artificial Intelligence Research, 46:165-201.

Dragomir R Radev, Mark Thomas Joseph, Bryan Gibson, and Pradeep Muthukrishnan. 2016. A bibliometric and network analysis of the field of computational linguistics. Journal of the Association for Information Science and Technology, 67(3):683-706.

James Ravenscroft, Maria Liakata, Amanda Clare, and Daniel Duma. 2017. Measuring scientific impact beyond academia: An assessment of existing impact metrics and proposed improvements. PloS one, 12(3):e0173152.

Natalie Schluter. 2018. The glass ceiling in NLP. In Proceedings of the 2018 Conference on Empirical Methods in Natural Language Processing, pages 2793-2798.

Simone Teufel, Advaith Siddharthan, and Dan Tidhar. 2006. Automatic classification of citation function. In Proceedings of the 2006 Conference on Empirical Methods in Natural Language Processing, pages 103-110.

Shaopeng Wu, Youbing Zhao, Farzad Parvinzamir, Nikolaos Th Ersotelos, Hui Wei, and Feng Dong. 2019. Literature explorer: effective retrieval of scientific documents through nonparametric thematic topic detection. The Visual Computer, pages 1-18.

Dani Yogatama, Michael Heilman, Brendan O'Connor, Chris Dyer, Bryan R Routledge, and Noah A Smith. 2011. Predicting a scientific community's response to an article. In Proceedings of the 2011 Conference on Empirical Methods in Natural Language Processing, pages 594-604.

Xiaodan Zhu, Peter Turney, Daniel Lemire, and André Vellino. 2015. Measuring academic influence: Not all citations are equal. Journal of the Association for Information Science and Technology, 66(2):408427.

\section{A Appendix}

Figures 6 through 20 (in the pages ahead) show example interactions with NLP Scholar that were discussed in Section 5. 


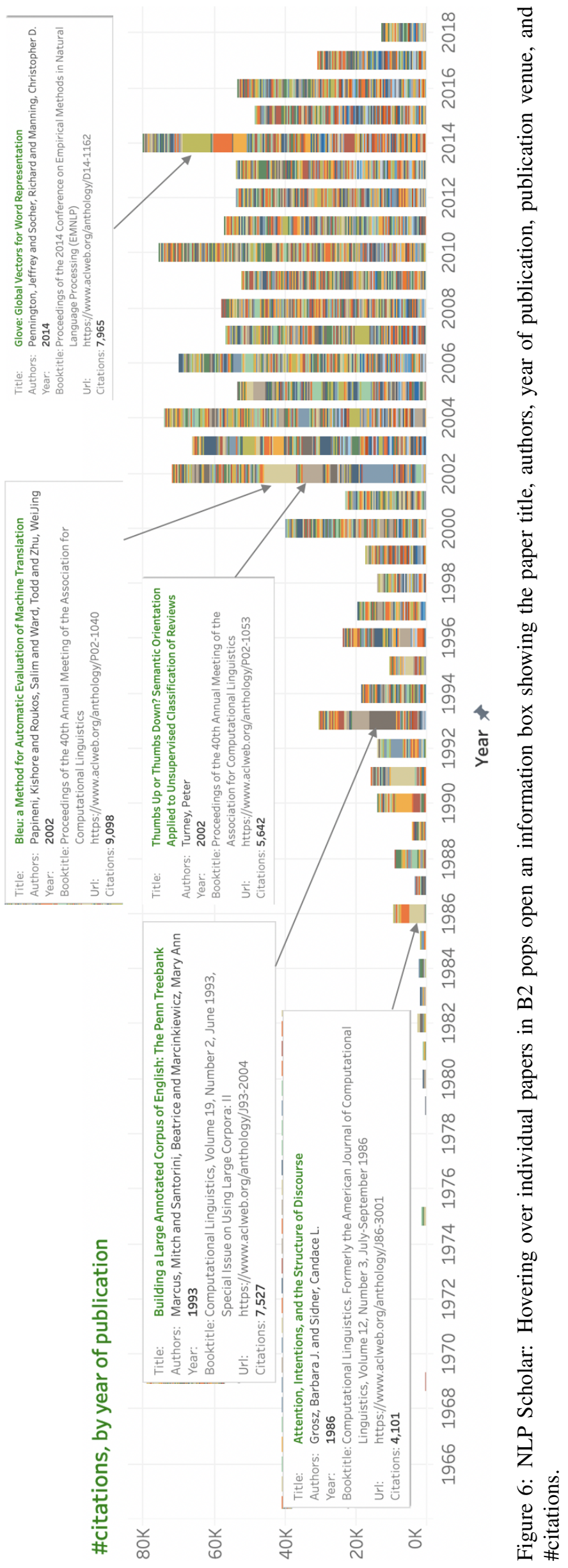



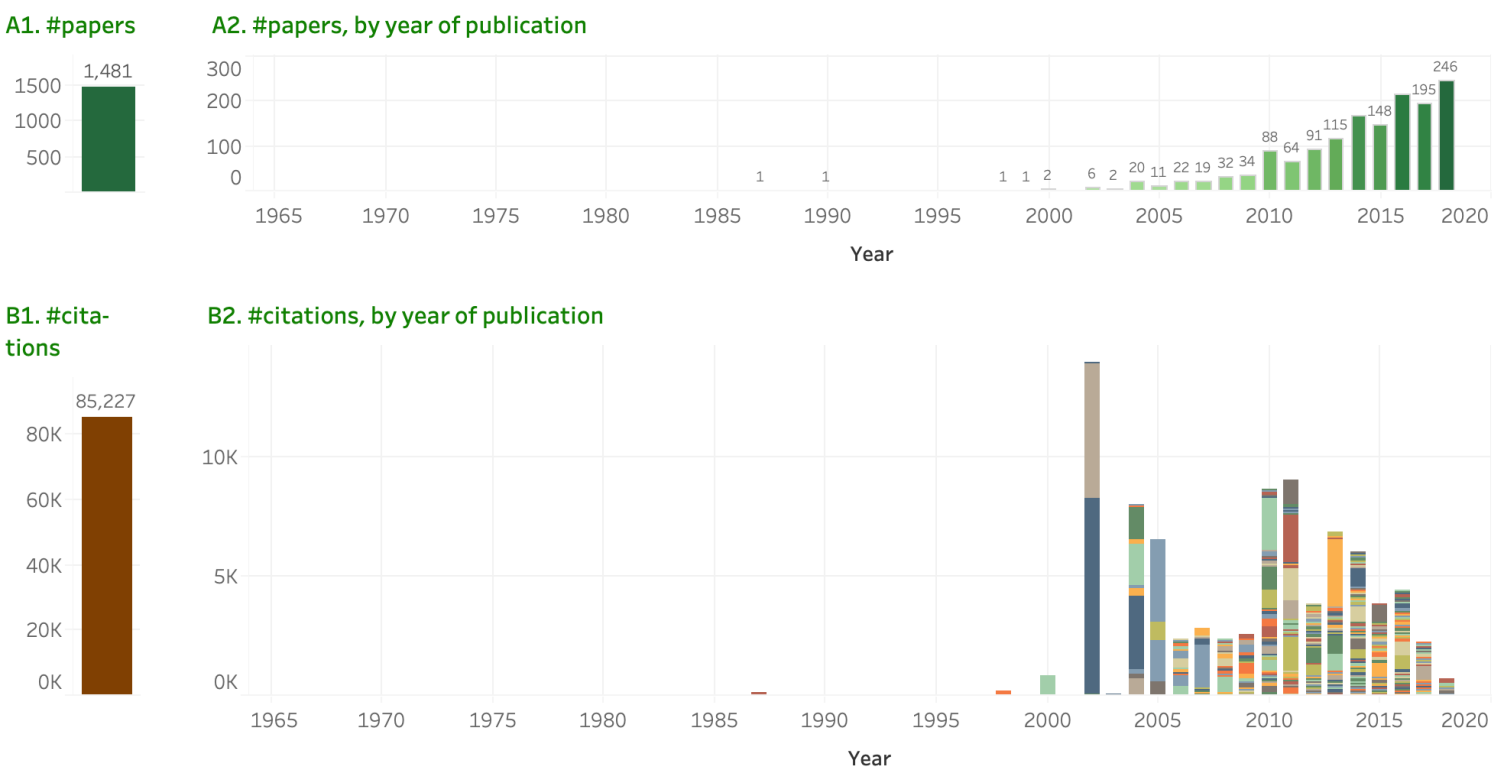

C. Papers

\begin{tabular}{|c|c|c|c|c|c|}
\hline Row & Paper-Id & Paper-Title & Author(s) & Year & Url \\
\hline 1 & W02-1011 & Thumbs up? Sentiment Classification using Machine Learning Techniques & Pang, Bo and Lee, Lillian and Vai... & 2002 & htt.. \\
\hline 2 & P02-1053 & Thumbs Up or Thumbs Down? Semantic Orientation Applied to Unsuper.. & Turney, Peter & 2002 & htt.. $\quad 5,642$ \\
\hline 3 & H05-1044 & Recognizing Contextual Polarity in Phrase-Level Sentiment Analysis & Wilson, Theresa and Wiebe, Jan.. & 2005 & htt.. $\quad 3,487$ \\
\hline 4 & P04-1035 & A Sentimental Education: Sentiment Analysis Using Subjectivity Summa.. & Pang, Bo and Lee, Lillian & 2004 & htt.. 3,109 \\
\hline 5 & D13-1170 & Recursive Deep Models for Semantic Compositionality Over a Sentimen.. & Socher, Richard and Perelygin, . & 2013 & htt.. $\quad 2,798$ \\
\hline 6 & L10-1-531 & SentiWordNet 3.0: An Enhanced Lexical Resource for Sentiment Analysi.. & Baccianella, Stefano and Esuli,.. & 2010 & htt.. $\quad 2,263$ \\
\hline 7 & J11-2001 & Lexicon-Based Methods for Sentiment Analysis & Taboada, Maite and Brooke, Jul.. & 2011 & htt.. $\square 1,982$ \\
\hline 8 & P05-1015 & Seeing Stars: Exploiting Class Relationships for Sentiment Categorizati.. & Pang, Bo and Lee, Lillian & 2005 & htt.. 1,743 \\
\hline 9 & P07-1056 & Biographies, Bollywood, Boom-boxes and Blenders: Domain Adaptation .. & Blitzer, John and Dredze, Mark .. & 2007 & htt.. 1,735 \\
\hline 10 & C04-1200 & Determining the Sentiment of Opinions & Kim, SooMin and Hovy, Eduard & 2004 & htt.. 1,723 \\
\hline
\end{tabular}

D. Authors

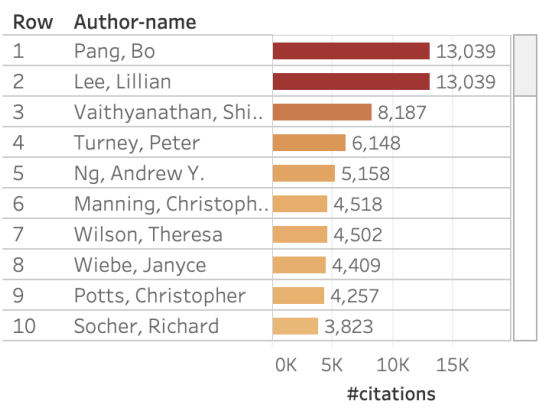

E. Search by year of publication, title term (unigram, bigram), or author name Year of publication 1965 to 2019

Unigram
\#emotional
affect
emotion
emotional
emotions
orientation
sentiment
stance
valence

Author Name

Bigram
Figure 7: NLP Scholar: After entering terms associated with sentiment analysis in the search box. 

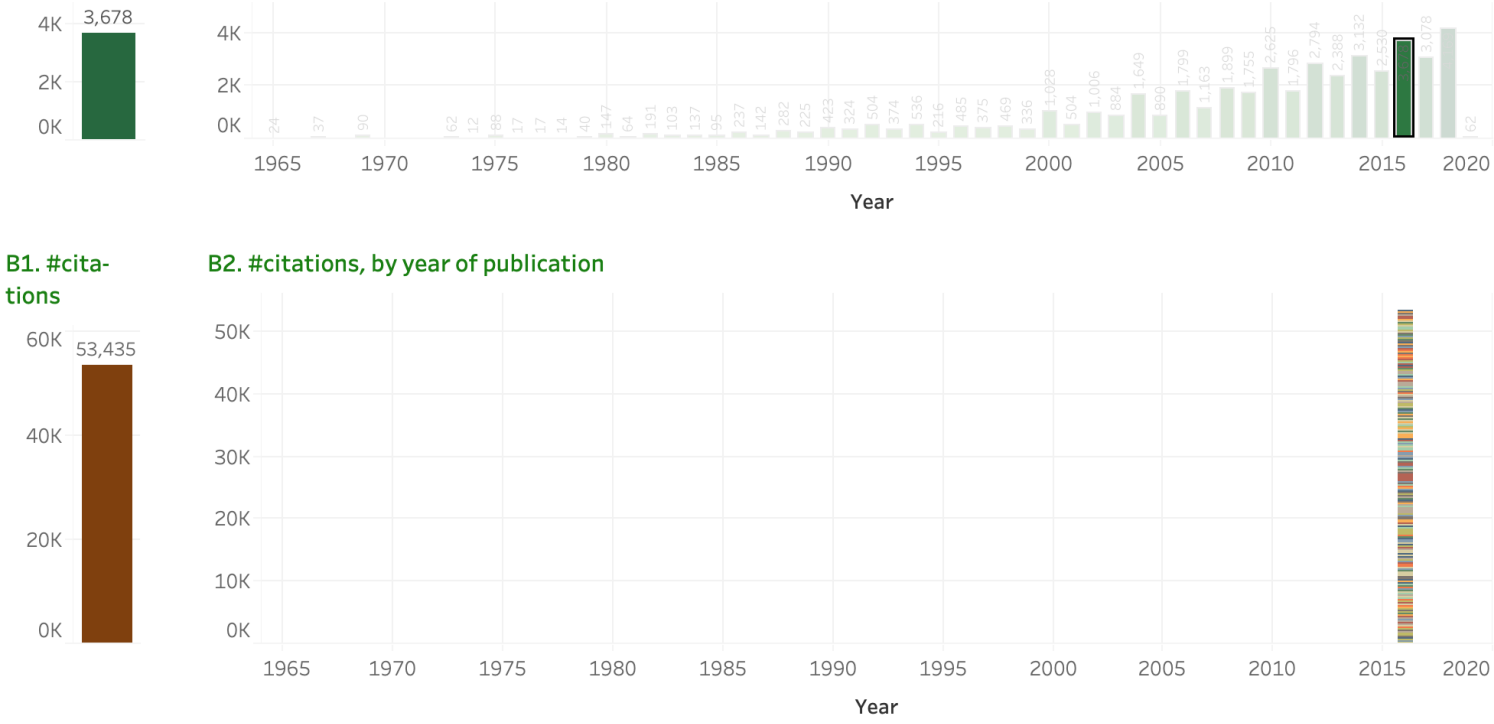

C. Papers

\begin{tabular}{|c|c|c|c|c|}
\hline Row & Paper-Id & Paper-Title & Author(s) & Url \\
\hline 1 & N16-3020 & "Why Should I Trust You?": Explaining the Predictions of Any Classifier & Ribeiro, Marco Tulio and Singh, .. 2016 & htt.. \\
\hline 2 & P16-1162 & Neural Machine Translation of Rare Words with Subword Units & Sennrich, Rico and Haddow, Bar.. 2016 & \begin{tabular}{l|l} 
htt.. & 1,028
\end{tabular} \\
\hline 3 & N16-1030 & Neural Architectures for Named Entity Recognition & Lample, Guillaume and Balleste.. 2016 & htt.. $\quad 957$ \\
\hline 4 & N16-1174 & Hierarchical Attention Networks for Document Classification & Yang, Zichao and Yang, Diyi and.. 2016 & htt... 952 \\
\hline 5 & D16-1264 & SQUAD: $100,000+$ Questions for Machine Comprehension of Text & Rajpurkar, Pranav and Zhang, Ji.. 2016 & htt.. $\quad 748$ \\
\hline 6 & P16-1101 & End-to-end Sequence Labeling via Bi-directional LSTM-CNNs-CRF & Ma, Xuezhe and Hovy, Eduard & htt.. $\square 611$ \\
\hline 7 & S16-1001 & SemEval-2016 Task 4: Sentiment Analysis in Twitter & Nakov, Preslav and Ritter, Alan .. 2016 & htt.. $\quad 567$ \\
\hline 8 & K16-1002 & Generating Sentences from a Continuous Space & Bowman, Samuel and Vilnis, Lu.. 2016 & htt.. $\square 561$ \\
\hline 9 & S16-1002 & SemEval-2016 Task 5: Aspect Based Sentiment Analysis & Pontiki, Maria and Galanis, Dim.. 2016 & htt.. 549 \\
\hline 10 & D16-1044 & Multimodal Compact Bilinear Pooling for Visual Question Answering an.. & Fukui, Akira and Park, Dong Huk.. 2016 & htt.. 430 \\
\hline
\end{tabular}

D. Authors

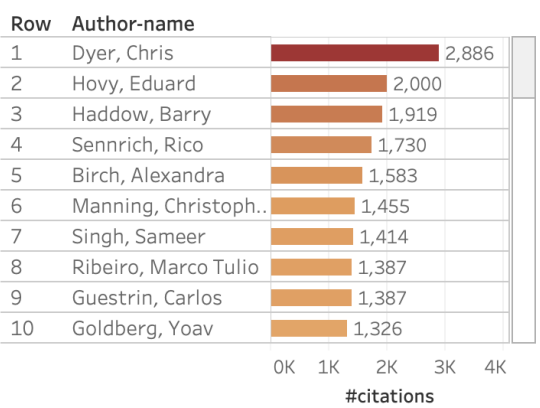

E. Search by year of publication, title term (unigram, bigram), or author name Year of publication

$\begin{array}{lll}1965 & D & \\ & & \\ & & \end{array}$

Figure 8: NLP Scholar: After clicking on the 2016 bar in the \#papers by year viz (A2). 
A1. \#papers A2. \#papers, by year of publication
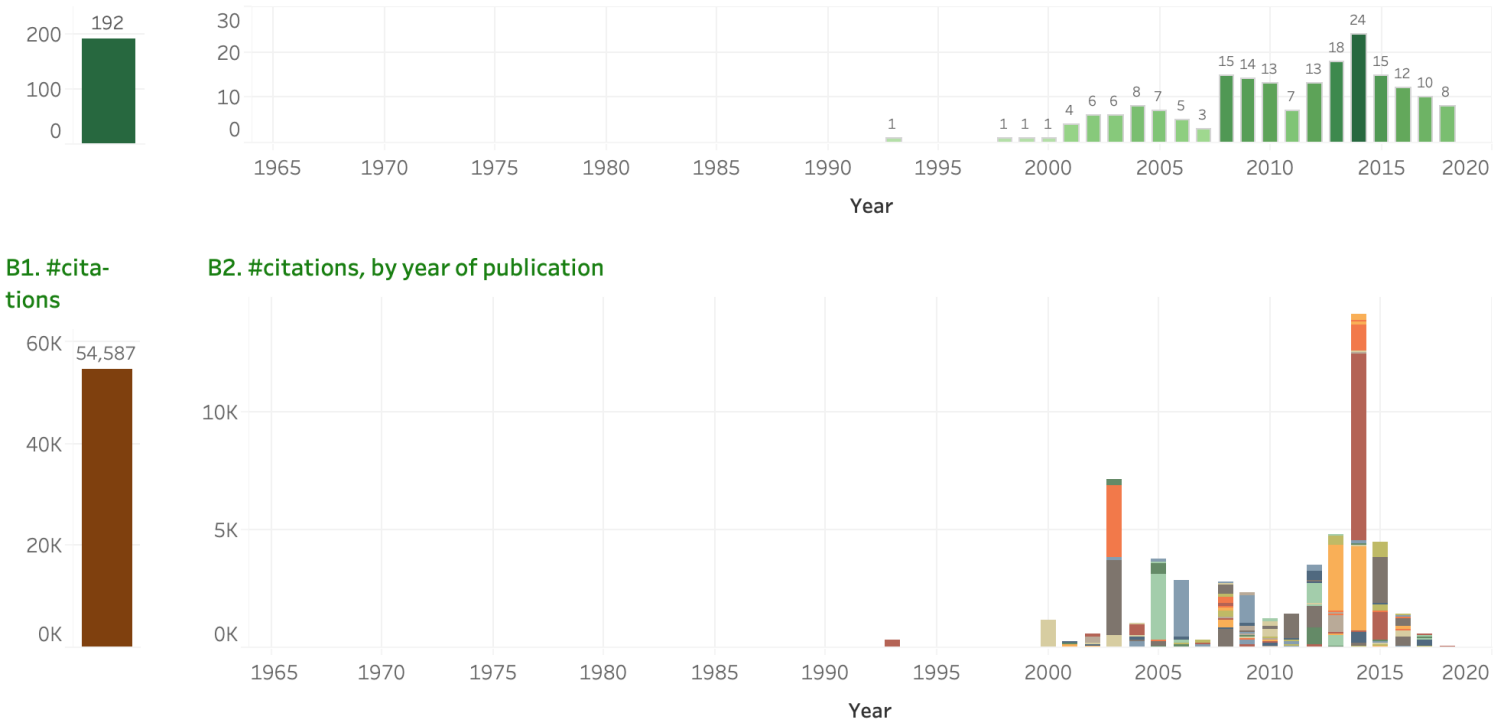

C. Papers

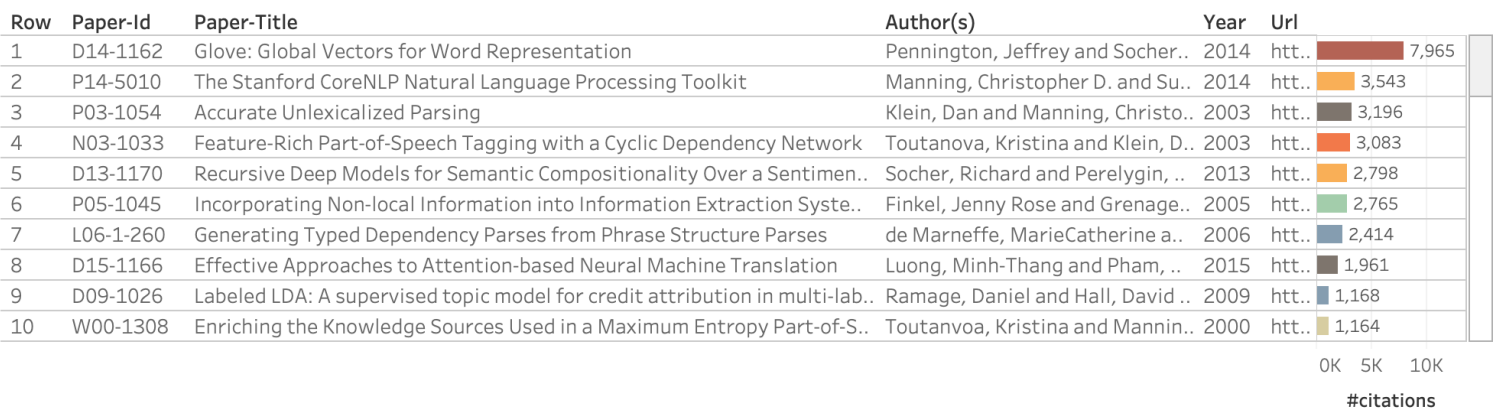

D. Authors

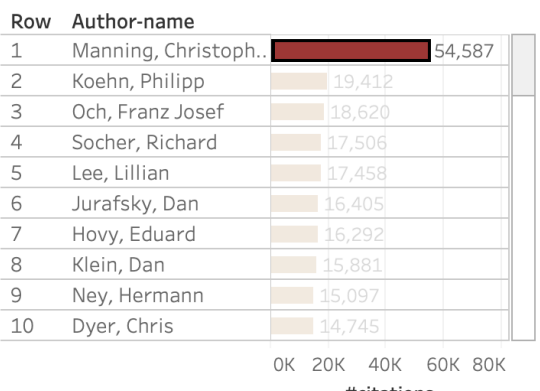

\#citations
E. Search by year of publication, title term (unigram, bigram), or author name Year of publication

1965

2019 $\begin{array}{lll}\text { Unigram } & \text { Bigram } & \text { Author Name }\end{array}$

Figure 9: NLP Scholar: After clicking on 'Manning, Christopher' in the Authors list (D). 


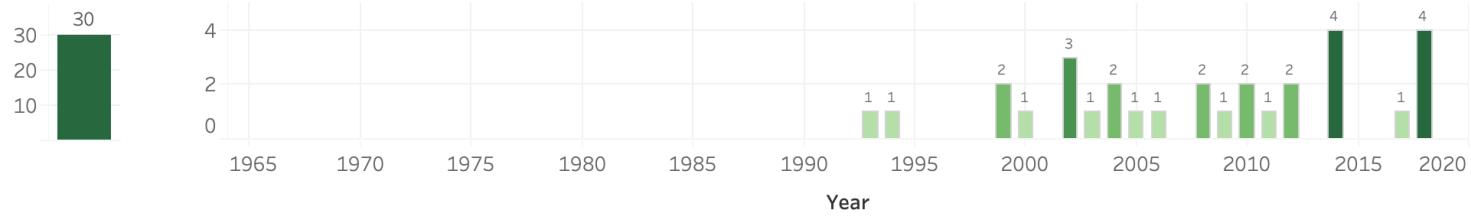

B1. \#cita- B2. \#citations, by year of publication

tions
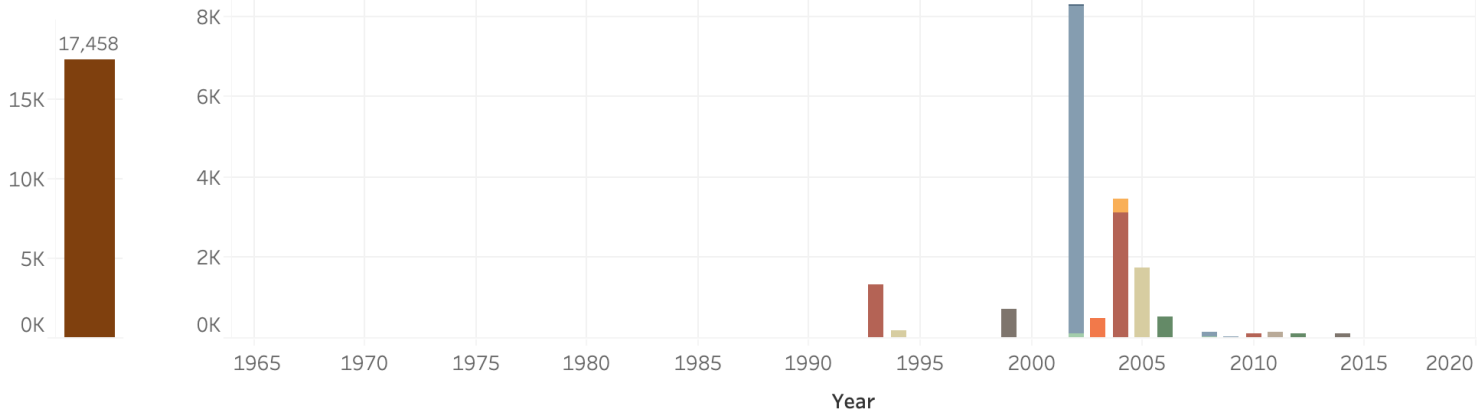

C. Papers

\begin{tabular}{|c|c|c|c|c|c|}
\hline Row & Paper-Id & Paper-Title & Author(s) & Year & Url \\
\hline 1 & W02-1011 & Thumbs up? Sentiment Classification using Machine Learning Techniques & Pang, Bo and Lee, Lillian and Vai.. & 2002 & htt.. \\
\hline 2 & P04-1035 & A Sentimental Education: Sentiment Analysis Using Subjectivity Summa.. & Pang, Bo and Lee, Lillian & 2004 & htt.. $\quad 3,109$ \\
\hline 3 & P05-1015 & Seeing Stars: Exploiting Class Relationships for Sentiment Categorizati.. & Pang, Bo and Lee, Lillian & 2005 & htt.. 1,743 \\
\hline 4 & P93-1024 & DISTRIBUTIONAL CLUSTERING OF ENGLISH WORDS & Pereira, Fernando and Tishby, N.. & 1993 & htt.. 1,322 \\
\hline 5 & P99-1004 & Measures of Distributional Similarity & Lee, Lillian & 1999 & htt..】 697 \\
\hline 6 & W06-1639 & Get out the vote: Determining support or opposition from Congressiona.. & Thomas, Matt and Pang, Bo and.. & 2006 & htt.. $\mid 536$ \\
\hline 7 & N03-1003 & Learning to Paraphrase: An Unsupervised Approach Using Multiple-Seq.. & Barzilay, Regina and Lee, Lillian & 2003 & htt.. I504 \\
\hline 8 & N04-1015 & Catching the Drift: Probabilistic Content Models, with Applications to G.. & Barzilay, Regina and Lee, Lillian & 2004 & htt.. | 341 \\
\hline 9 & P94-1038 & Similarity-Based Estimation of Word Cooccurrence Probabilities & Dagan, Ido and Pereira, Fernan.. & 1994 & htt.. 183 \\
\hline 10 & W11-0609 & Chameleons in Imagined Conversations: A New Approach to Understand.. & DanescuNiculescuMizil, Cristia.. & 2011 & htt.. 147 \\
\hline
\end{tabular}

10 W11-0609 Chameleons in Imagined Conversations: A New Approach to Understand.. DanescuNiculescuMlizil, Cristia.. 2011 htt.. 147 $0 \mathrm{~K} 5 \mathrm{~K} 10 \mathrm{~K}$

\#citations

D. Authors

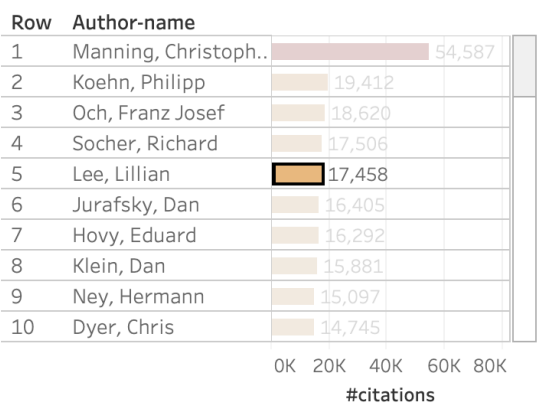

E. Search by year of publication, title term (unigram, bigram), or author name Year of publication

$$
1965
$$

Unigram

Uram

Bigram

Author Name

Figure 10: NLP Scholar: After clicking on 'Lee, Lillian' in the Authors list (D). 

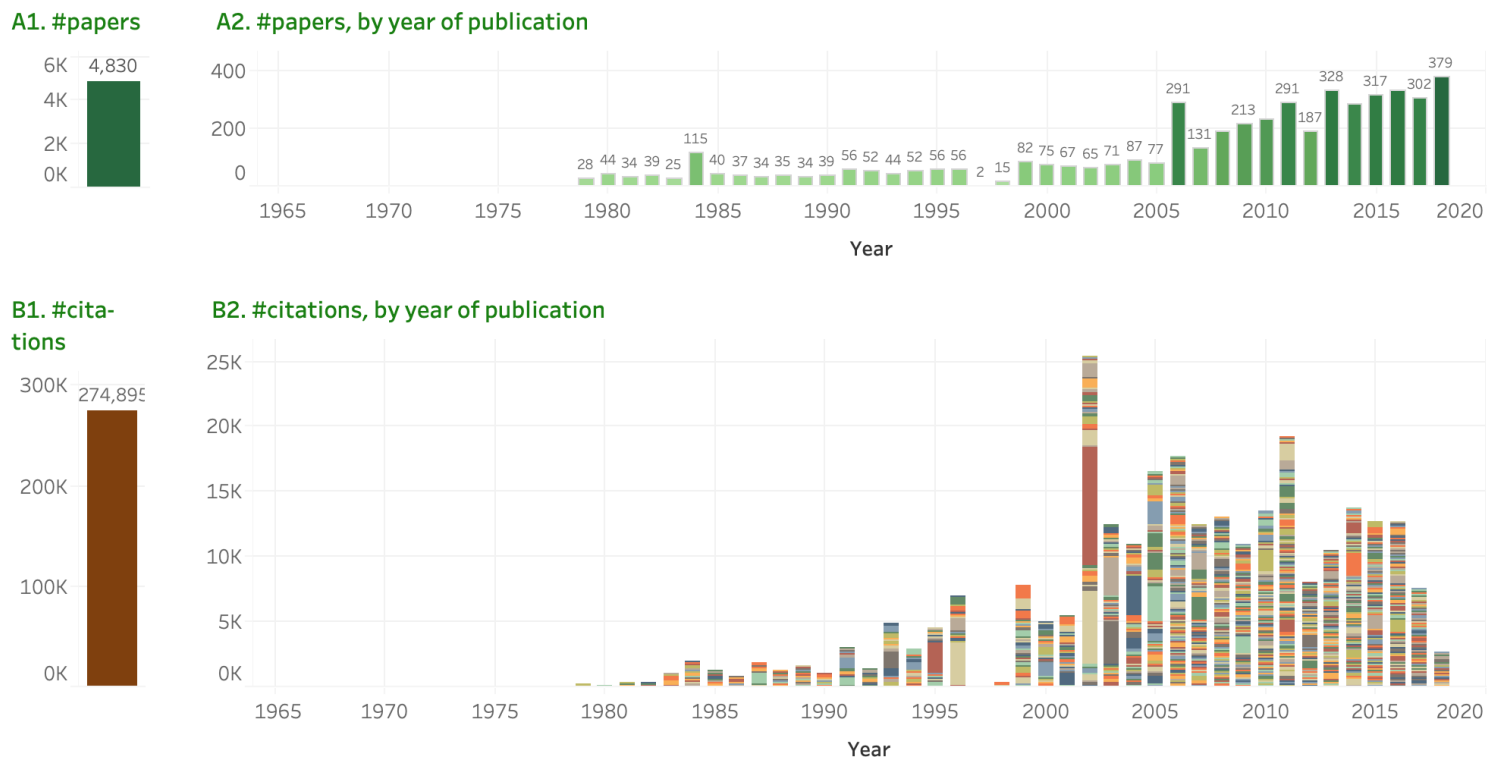

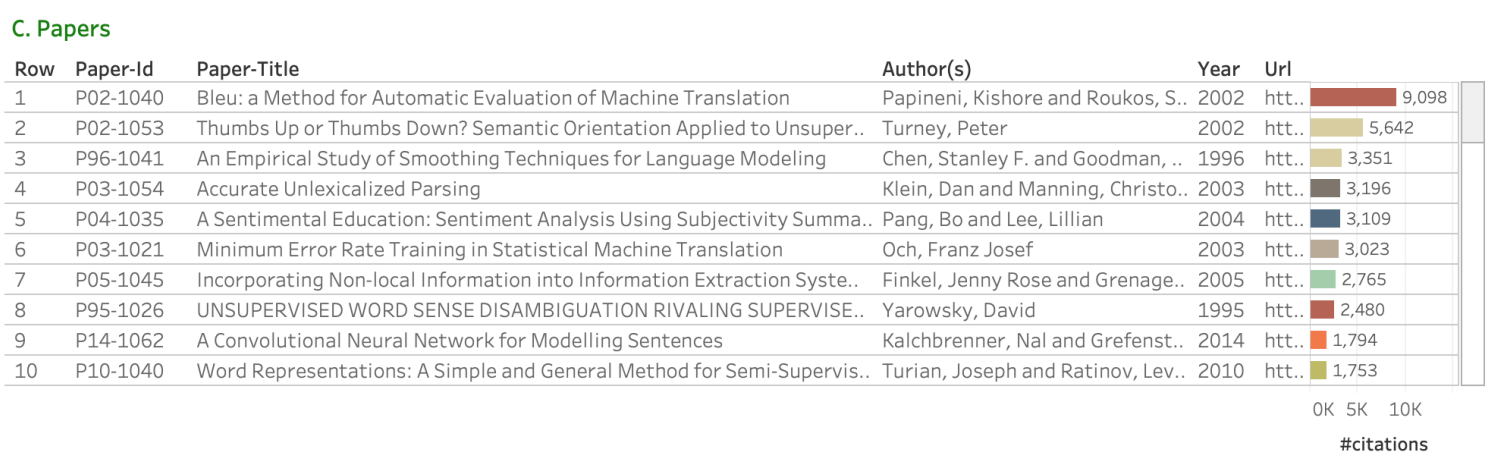

\section{Authors}

Row Author-name

1 Manning, Christoph

2 Roukos, Salim

3 Papineni, Kishore

$4 \quad$ Zhu, WeiJing

5 Ward, Todd

Lee, Lillian

$7 \quad$ Klein, Dan

Och, Franz Josef

9 Turney, Peter

10 Pang, Bo

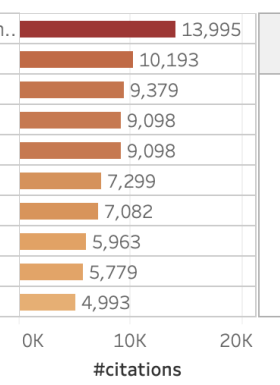

E. Search by year of publication, title term (unigram, bigram), or author name Year of publication

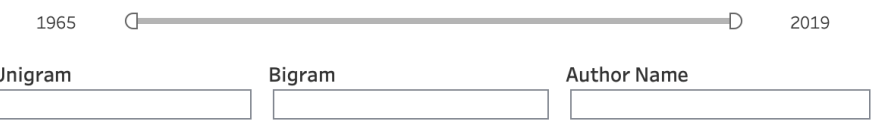

F1. Venue and Paper Type

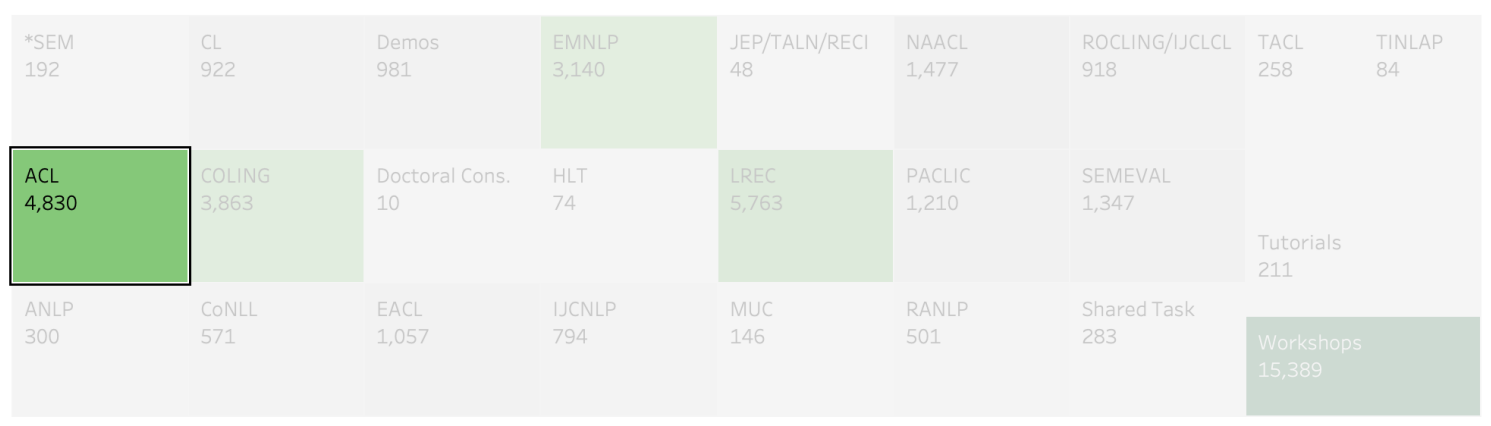

Figure 11: NLP Scholar: After clicking on 'ACL' in the venue and paper type treemap (F1). 

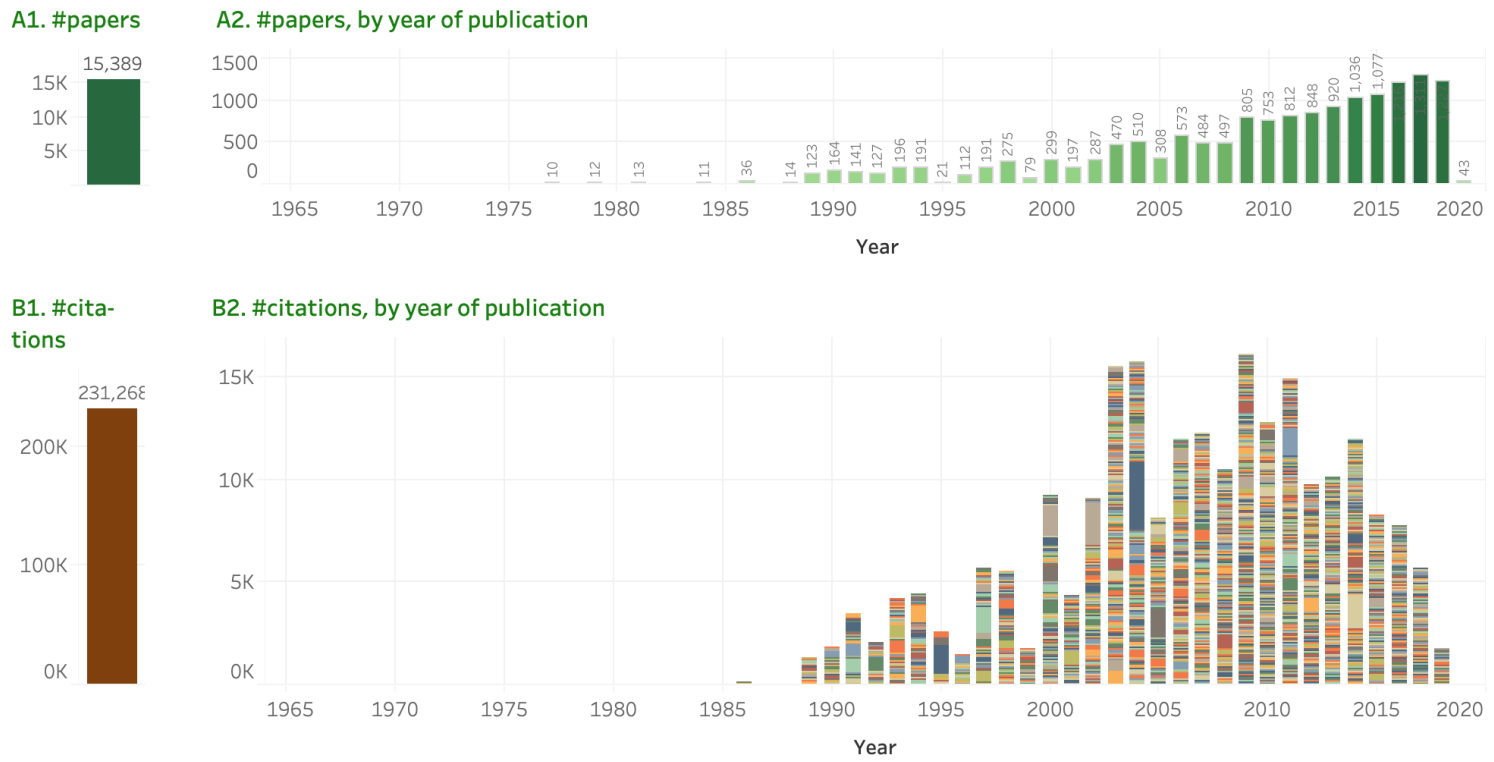

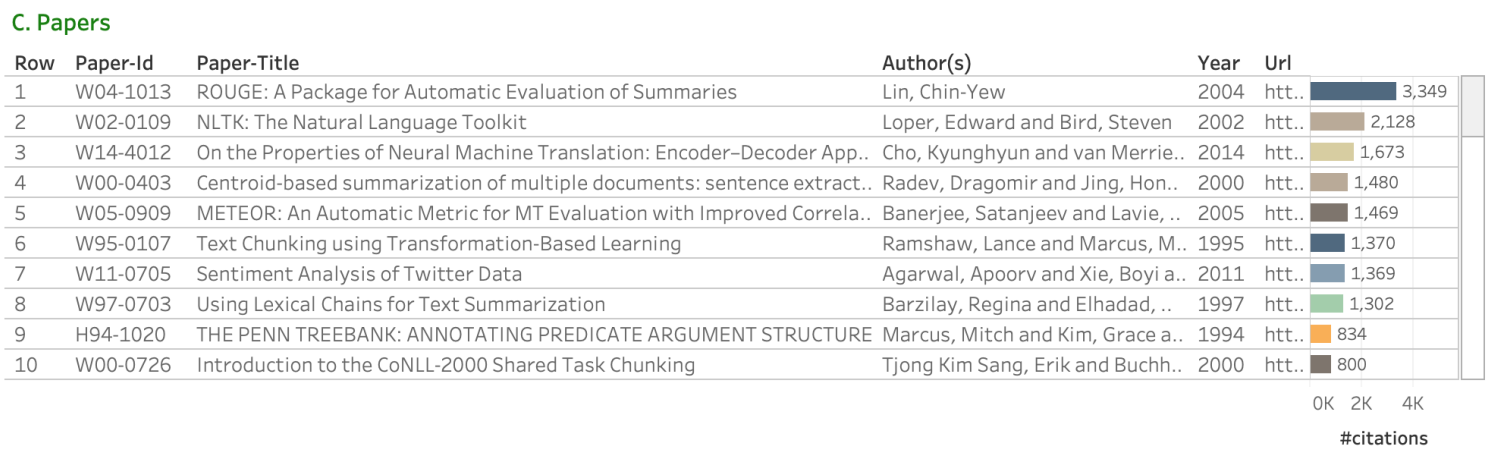

\section{Authors}

Row Author-name

1 Koehn, Philipp

2 Monz, Christof

3 Manning, Christoph.

4 Lavie, Alon

5 Lin, Chin-Yew

CallisonBurch, Chris

Marcus, Mitch

Palmer, Martha

Specia, Lucia

Rambow, Owen

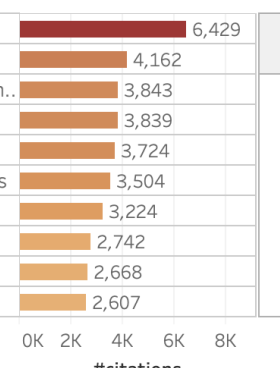

\#citations
E. Search by year of publication, title term (unigram, bigram), or author name Year of publication

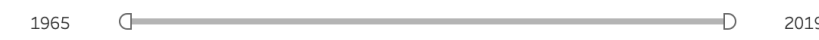

$\begin{array}{lll}\text { Unigram } & \text { Bigram } & \text { Author Name }\end{array}$

F1. Venue and Paper Type

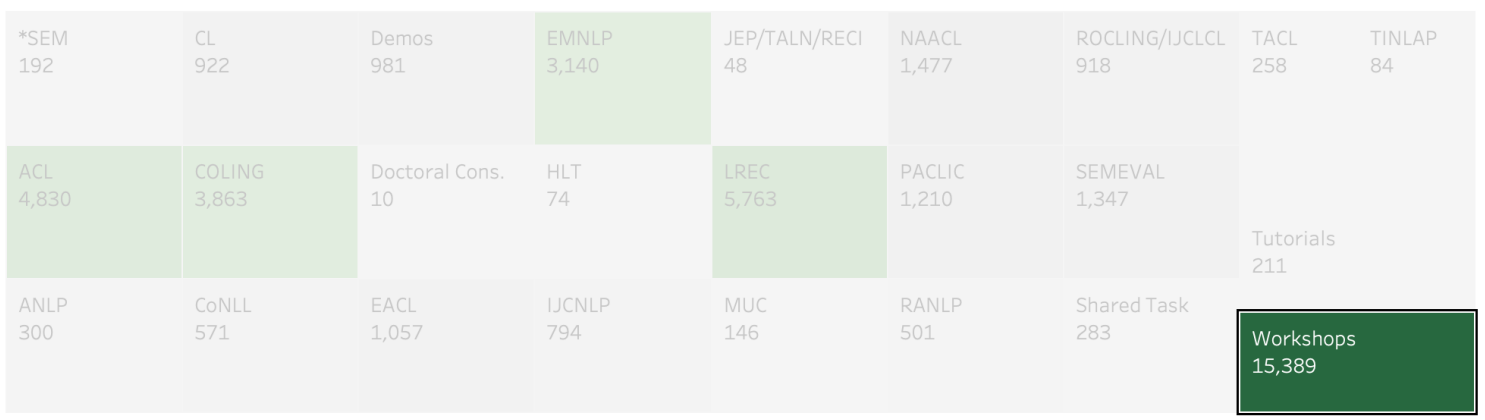

Figure 12: NLP Scholar: After clicking on 'Workshops' in the venue and paper type treemap (F1). 

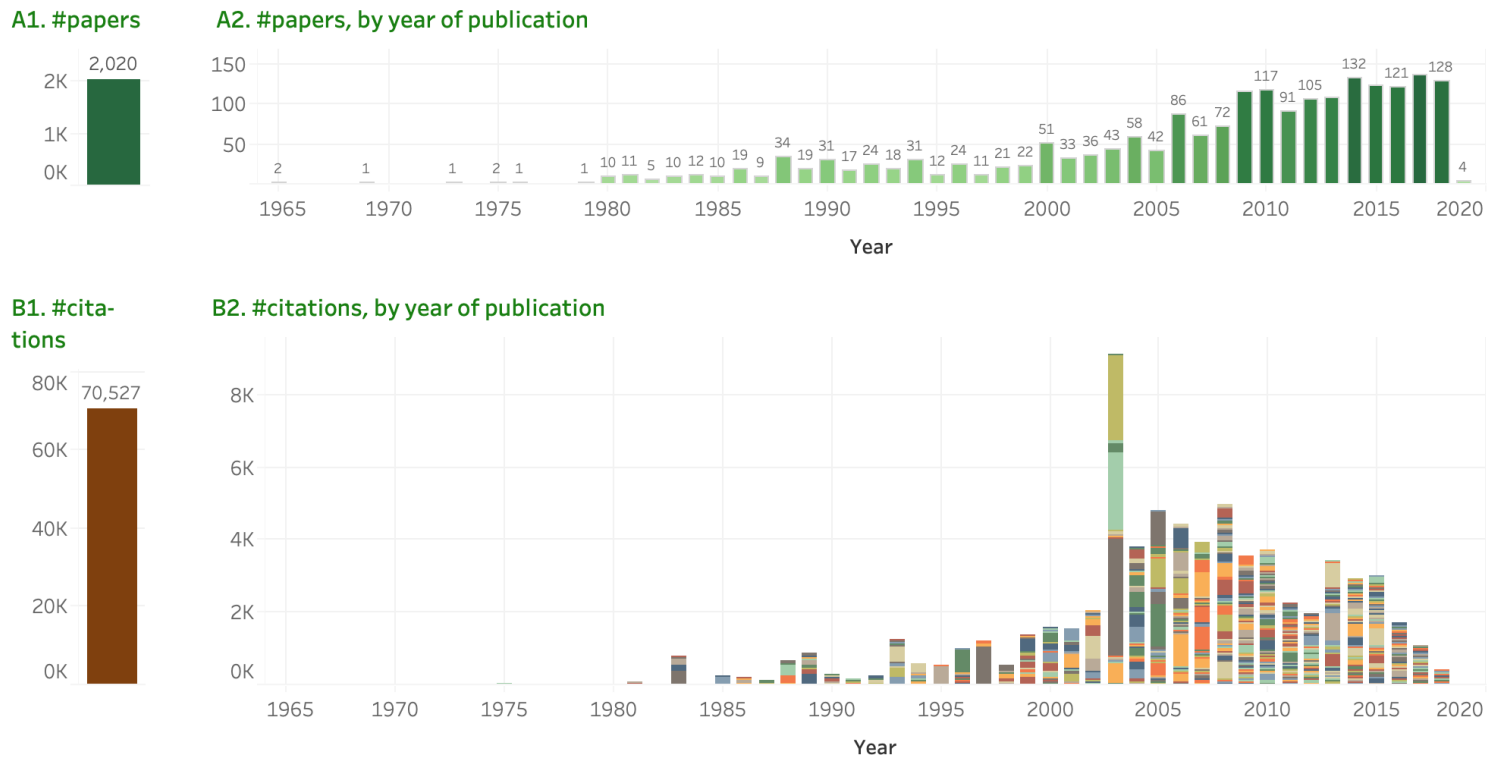

\section{Papers}

Row Paper-Id

Author(s)

Year Url

Klein, Dan and Manning, Christo.. 2003 htt.

N03-1028 Shallow Parsing with Conditional Random Fields $\quad$ Sha, Fei and Pereira, Fernando 2003 ht

P05-1022 Coarse-to-Fine n-Best Parsing and MaxEnt Discriminative Reranking Charniak, Eugene and Johnson, .. 2005 htt.. 1.184

J97-3002 Stochastic Inversion Transduction Grammars and Bilingual Parsing of P. . Wu, Dekai $\quad 1997$ htt.. 1,016

W06-2920 CoNLL-X Shared Task on Multilingual Dependency Parsing $\quad$ Buchholz, Sabine and Marsi, Er.. 2006 htt.. 911

H05-1066 Non-Projective Dependency Parsing using Spanning Tree Algorithms McDonald, Ryan and Pereira, Fe.. 2005 htt.. 905

105-1003 Discriminative Reranking for Natural Language Parsing Collins, Michael and Koo, Terry 2005 htt... 832

P13-1045 Parsing with Compositional Vector Grammars $\quad$ Socher, Richard and Bauer, Joh.. 2013 htt.. 748

10 D07-1096 The CoNLL 2007 Shared Task on Dependency Parsing Nivre, Joakim and Hall, Johan a.. 2007 htt.. 699

\section{OK $2 \mathrm{~K} \quad 4 \mathrm{~K}$}

\#citations

D. Authors

Row Author-name

$1 \quad$ Nivre, Joakim

2 Manning, Christoph.

Collins, Michael

Klein, Dan

Johnson, Mark

Pereira, Fernando

Charniak, Eugene

McDonald, Ryan

Nilsson, Jens

Hall, Johan

E. Search by year of publication, title term (unigram, bigram), or author name Year of publication

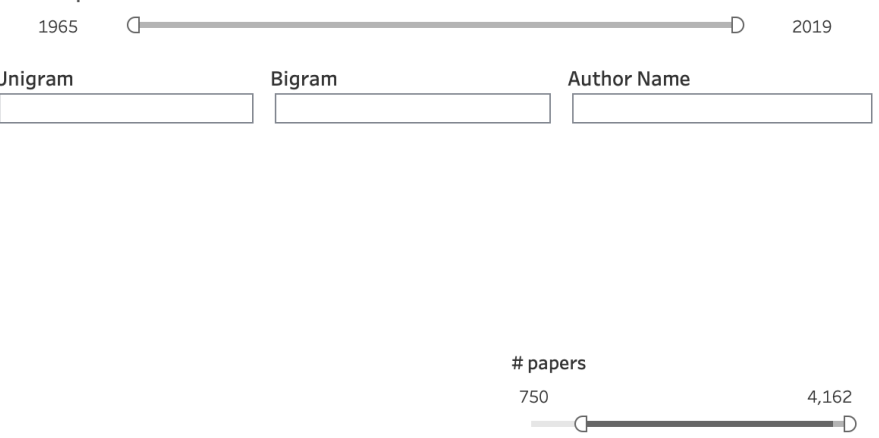

F2. Title Unigrams

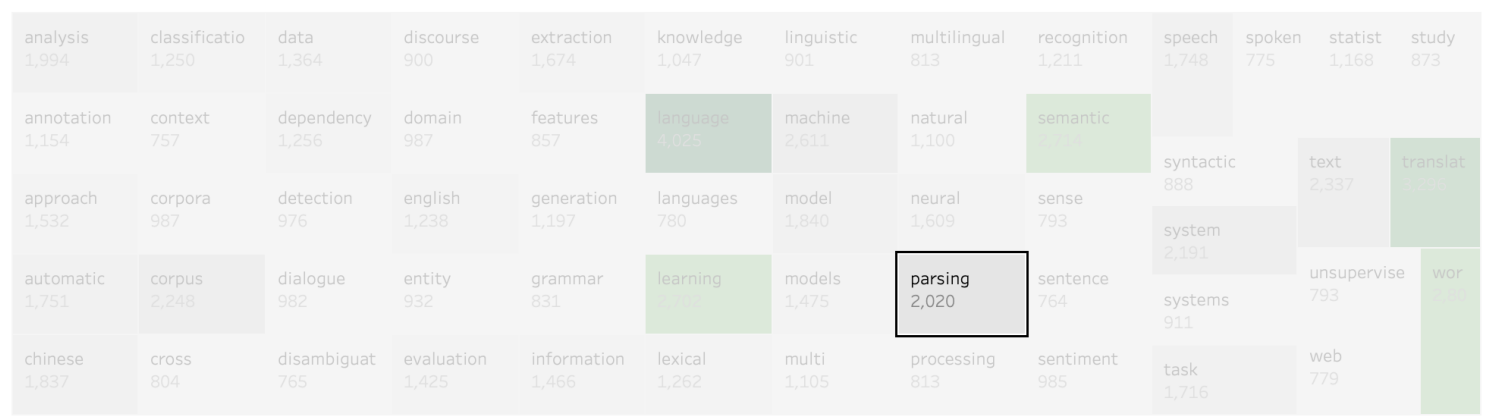

Figure 13: NLP Scholar: After clicking on 'parsing' in the unigrams treemap (F2) 

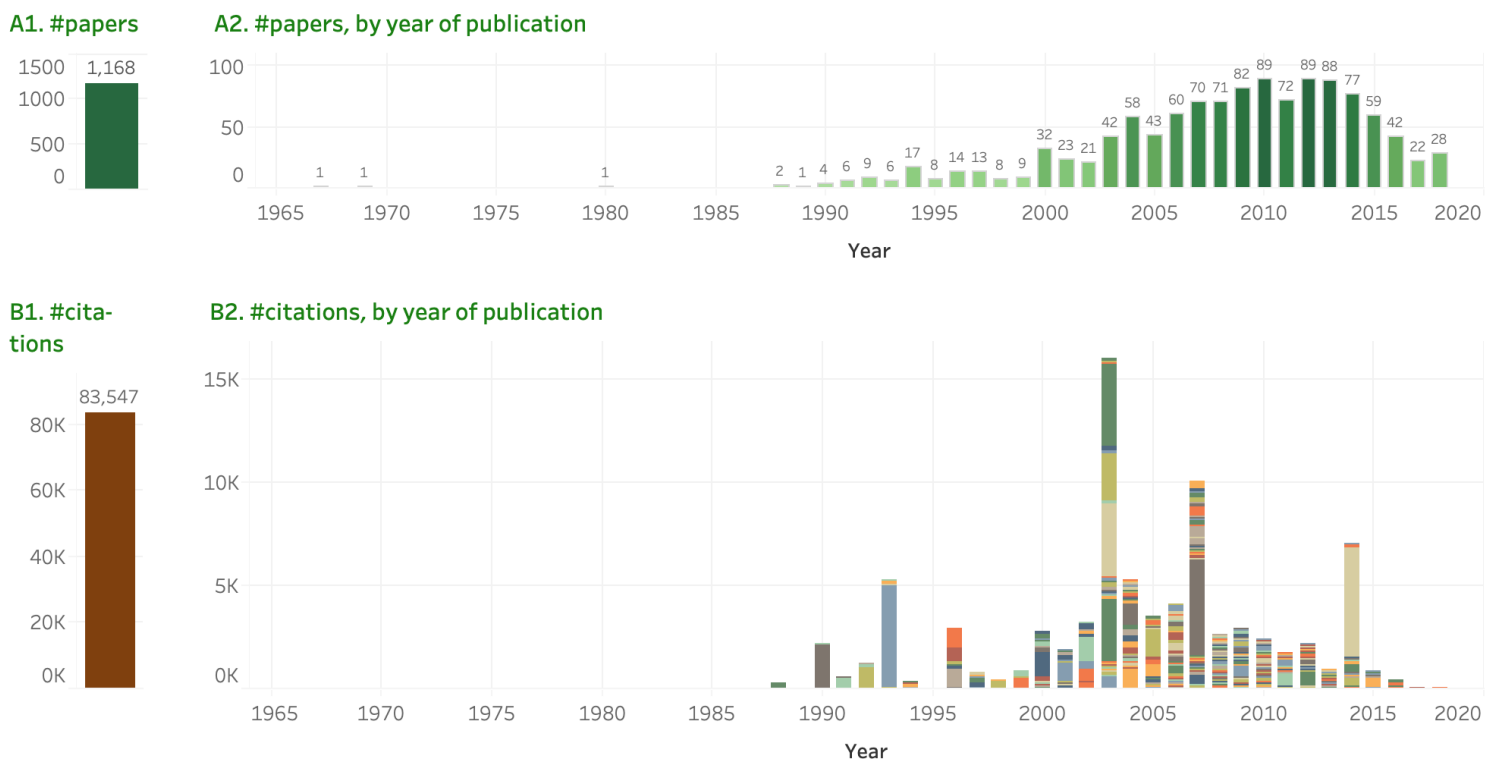

\begin{tabular}{|c|c|c|c|c|c|}
\hline Row & Paper-Id & Paper-Title & Author(s) & Year & Url \\
\hline 1 & D14-1179 & Learning Phrase Representations using RNN Encoder-Decoder for Stati.. & Cho, Kyunghyun and van Merrie.. & 2014 & htt.. $\quad 5,344$ \\
\hline 2 & J93-2003 & The Mathematics of Statistical Machine Translation: Parameter Estima.. & Brown, Peter F. and Della Pietra.. & 1993 & htt.. $\quad 5,047$ \\
\hline 3 & P07-2045 & Moses: Open Source Toolkit for Statistical Machine Translation & Koehn, Philipp and Hoang, Hieu . & 2007 & htt.. 4,581 \\
\hline 4 & J03-1002 & A Systematic Comparison of Various Statistical Alignment Models & Och, Franz Josef and Ney, Herm.. & 2003 & htt.. $\quad 4,040$ \\
\hline 5 & N03-1017 & Statistical Phrase-Based Translation & Koehn, Philipp and Och, Franz J.. & 2003 & htt.. $\quad 3,501$ \\
\hline 6 & P03-1021 & Minimum Error Rate Training in Statistical Machine Translation & Och, Franz Josef & 2003 & htt.. 3,023 \\
\hline 7 & $103-4003$ & Head-Driven Statistical Models for Natural Language Parsing & Collins, Michael & 2003 & htt.. $\quad 2,271$ \\
\hline 8 & J90-2002 & A Statistical Approach to Machine Translation & Brown, Peter F. and Cocke, John.. & 1990 & htt.. $\square 2,102$ \\
\hline 9 & P05-1033 & A Hierarchical Phrase-Based Model for Statistical Machine Translation & Chiang, David & 2005 & htt.. 1,288 \\
\hline 10 & P02-1038 & Discriminative Training and Maximum Entropy Models for Statistical M.. & Och, Franz Josef and Ney, Herm.. & 2002 & htt.. 1,240 \\
\hline
\end{tabular}

\section{Authors}

Row Author-name

1 Och, Franz Josef

2 Koehn Philipp

$3 \quad$ Ney, Hermann

4 Brown, Peter F.

5 Mercer, Robert L.

Della Pietra, Vincen.

Della Pietra, Stephe..

CallisonBurch, Chris

Schwenk, Holger

10 Zens, Richard

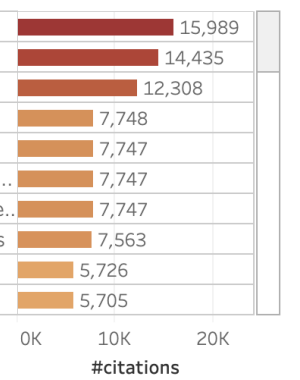

F2. Title Unigrams

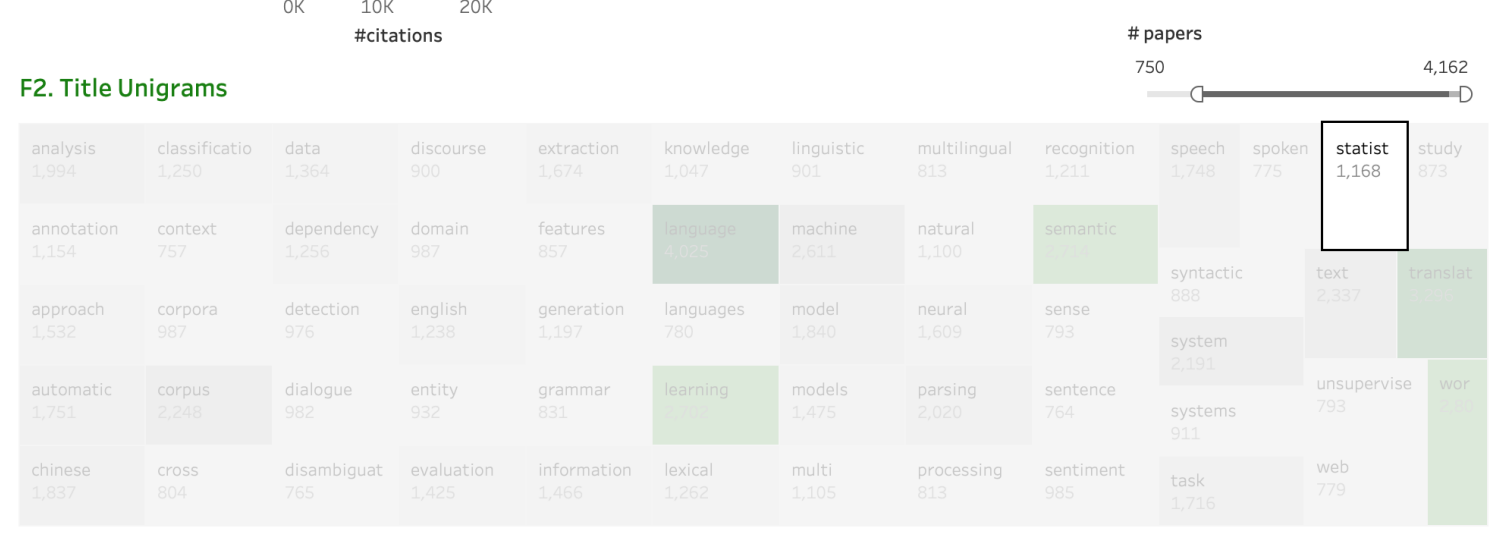

E. Search by year of publication, title term (unigram, bigram), or author name Year of publication

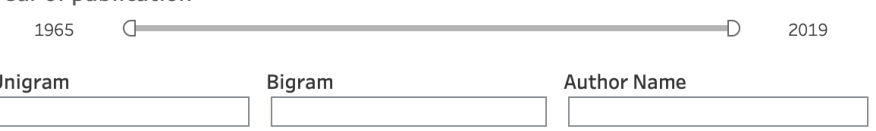

Figure 14: NLP Scholar: After clicking on 'statistical' in the unigrams treemap (F2). 


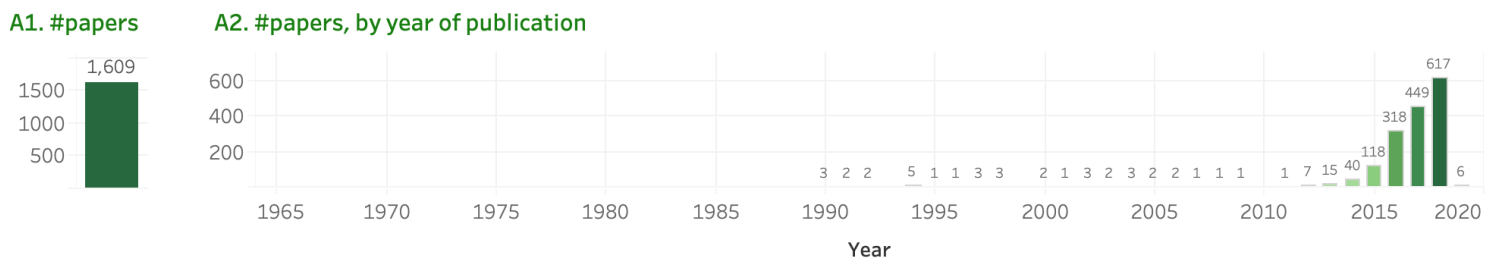

B1. \#cita- $\quad$ B2. \#citations, by year of publication

tions

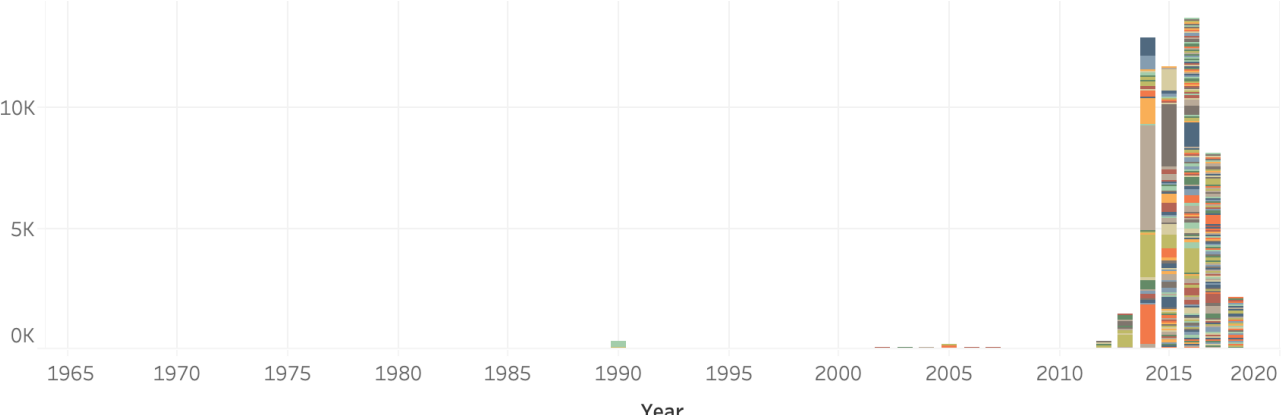

\section{Papers}

Row Paper-Id Paper-Title

14-1181 Convolutional Neural Networks for Sentence Classification $\quad$ Kim, Yoon 2014 htt.

D15-1166 Effective Approaches to Attention-based Neural Machine Translation ～Luong, Minh-Thang and Pham, .. 2015 htt.. 1.961

P14-1062 A Convolutional Neural Network for Modelling Sentences $\quad$ Kalchbrenner, Nal and Grefenst.. 2014 htt.. 1,794

W14-4012 On the Properties of Neural Machine Translation: Encoder-Decoder App.. Cho, Kyunghyun and van Merrie.. 2014 htt.. 1,673

D14-1082 A Fast and Accurate Dependency Parser using Neural Networks $\quad$ Chen, Dangi and Manning, Chris. 2014 htt.. 1,110

P16-1162 Neural Machine Translation of Rare Words with Subword Units $\quad$ Sennrich, Rico and Haddow, Bar.. 2016 htt.. 1,028

N16-1030 Neural Architectures for Named Entity Recognition $\quad$ Lample, Guillaume and Balleste.. 2016 htt.. 957

D15-1044 A Neural Attention Model for Abstractive Sentence Summarization $\quad$ Rush. Alexander M. and Chopra,.. 2015 htt.. 910

C14-1008 Deep Convolutional Neural Networks for Sentiment Analysis of Short Te.. dos Santos, Cicero and Gatti, M.. 2014 htt.. 697

0 D15-1167 Document Modeling with Gated Recurrent Neural Network for Sentime.. Tang, Duyu and Qin, Bing and Li.. 2015 htt.. \606

\#citations

\section{Authors}

Row Author-name

1 Kim, Yoon

2 Manning, Christoph

3. Cho, Kyunghyun

$4 \quad$ Luong, Minh-Thang

Bengio, Yoshua

Sennrich, Rico

Blunsom, Phil

Haddow, Barry

Kalchbrenner, $\mathrm{Nal}$

10 Pham, Hieu

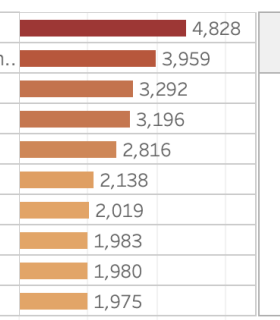

$0 \mathrm{~K} \quad 2 \mathrm{~K} \quad 4 \mathrm{~K}$
\#citations

F2. Title Unigrams

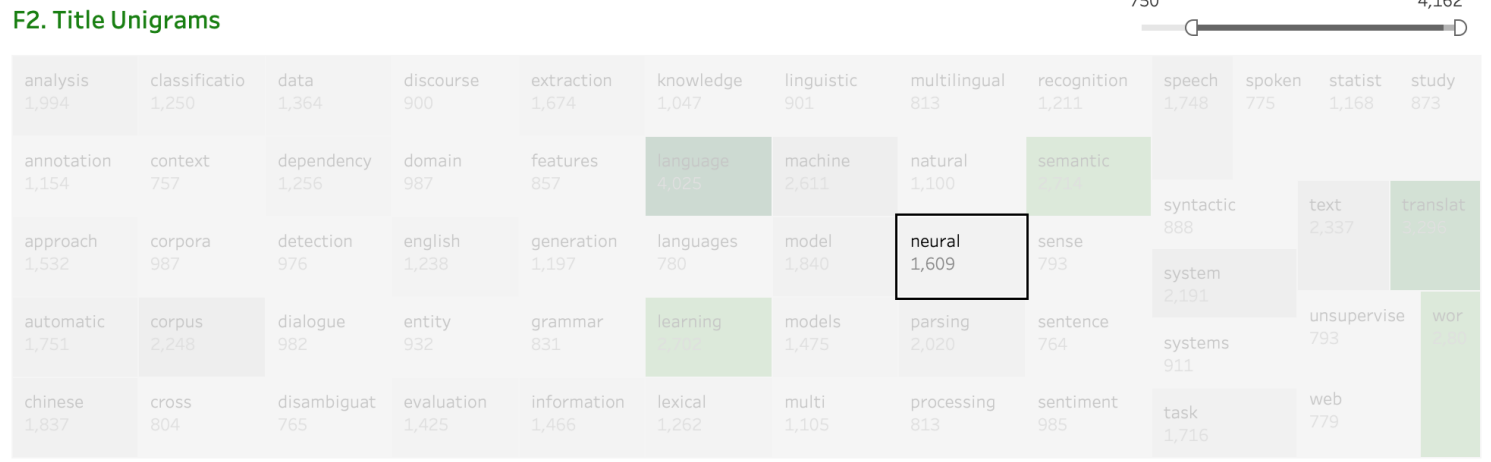

E. Search by year of publication, title term (unigram, bigram), or author name Year of publication

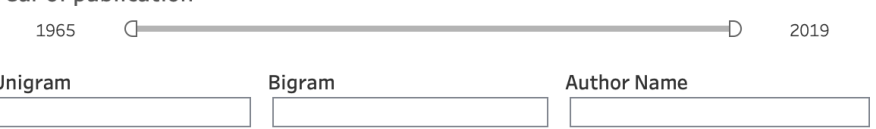

\# papers

750

Figure 15: NLP Scholar: After clicking on 'neural' in the unigrams treemap (F2). 

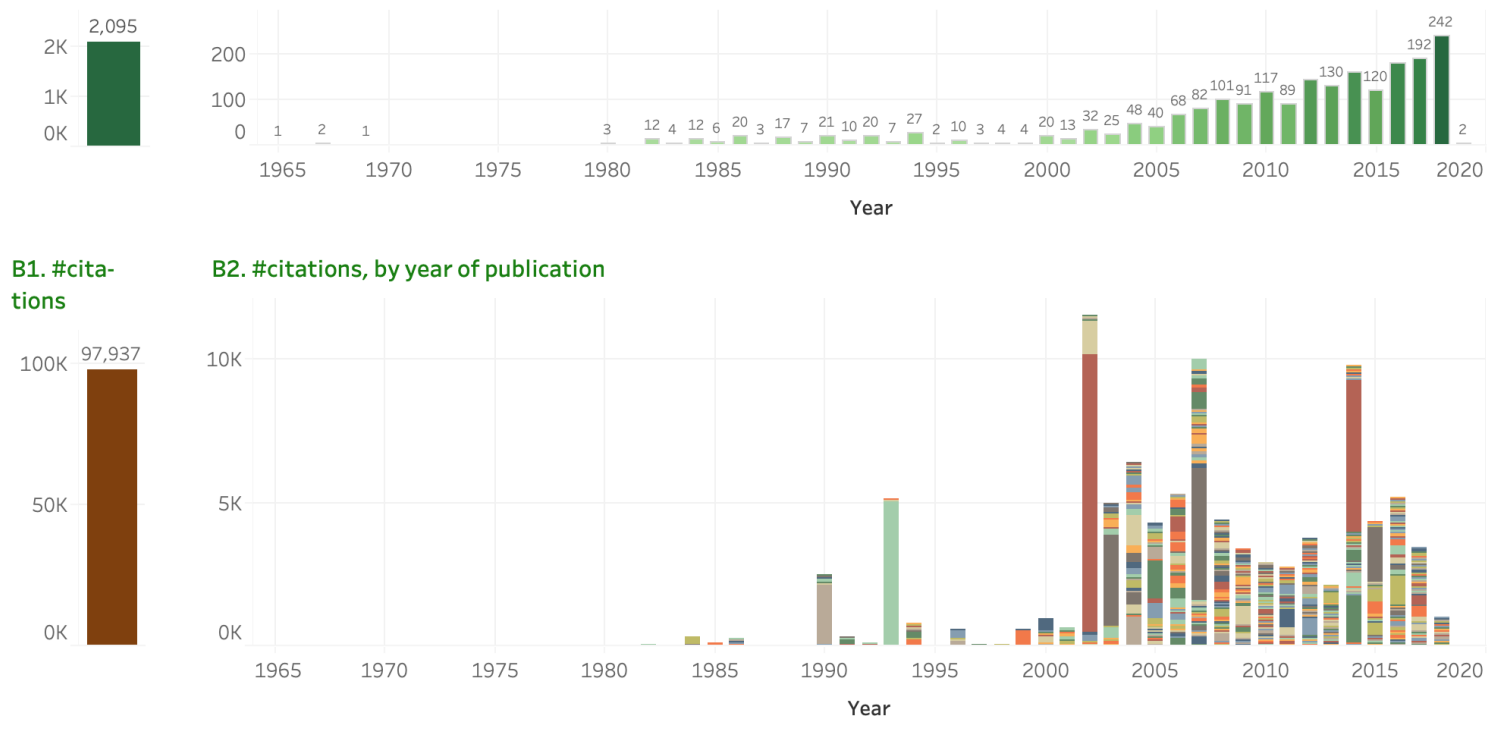

\section{Papers}

Row Paper-1d

P02-1040 Bleu: a Method for Automatic Evaluationor(s) Author Url

D14-1179 Learning Phrase Representations using RNN Encoder-Decoder for Stati.. Cho, Kyunghyun and van Merrie.. 2014 htt.

J93-2003 The Mathematics of Statistical Machine Translation: Parameter Estima.. Brown, Peter F. and Della Pietra.. 1993 htt.. 5,047

P07-2045 Moses: Open Source Toolkit for Statistical Machine Translation $\quad$ Koehn, Philipp and Hoang, Hieu .. 2007 htt.. 4,581

P03-1021 Minimum Error Rate Training in Statistical Machine Translation $\quad$ Och, Franz Josef $\quad 2003$ htt.. 3,023

J90-2002 A Statistical Approach to Machine Translation Brown, Peter F. and Cocke, John.. 1990 htt.. 2,102

D15-1166 Effective Approaches to Attention-based Neural Machine Translation Luong, Minh-Thang and Pham,.. 2015 htt.. 1,961

W14-4012 On the Properties of Neural Machine Translation: Encoder-Decoder App.. Cho, Kyunghyun and van Merrie.. 2014 htt.. 1,673

P05-1033 A Hierarchical Phrase-Based Model for Statistical Machine Translation Chiang, David $\quad 2005$ htt..】 1,288

0 P02-1038 Discriminative Training and Maximum Entropy Models for Statistical M.. Och, Franz Josef and Ney, Herm.. 2002 htt.. 1,240

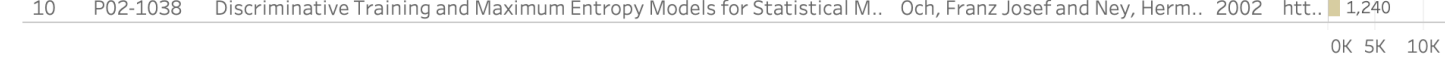

\#citations

\section{Authors}

\begin{tabular}{ll} 
Row & Author-name \\
\hline 1 & Koehn, Philipp \\
\hline 2 & Papineni, Kishore \\
\hline 3 & Roukos, Salim \\
\hline 4 & Ward, Todd \\
\hline 5 & Zhu, WeiJing \\
\hline 6 & Och, Franz Josef \\
\hline 7 & CallisonBurch, Chris \\
\hline 8 & Cho, Kyunghyun \\
\hline 9 & Bengio, Yoshua \\
\hline 10 & Brown, Peter F. \\
\hline
\end{tabular}

E. Search by year of publication, title term (unigram, bigram), or author name Year of publication

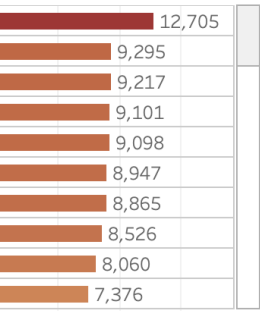

OK $5 \mathrm{~K} \quad 10 \mathrm{~K} \quad 15 \mathrm{~K}$ \#citations
Unigram

1965

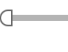

F3. Title Bigrams

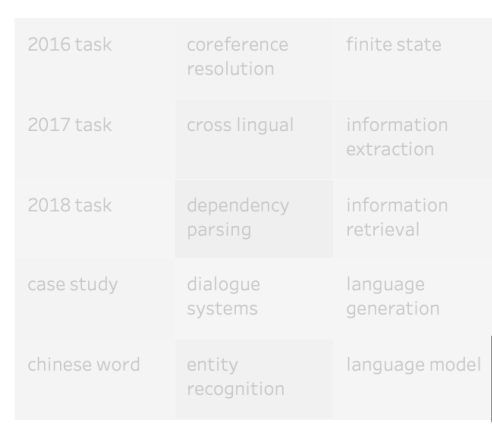


A1. \#papers A2. \#papers, by year of publication
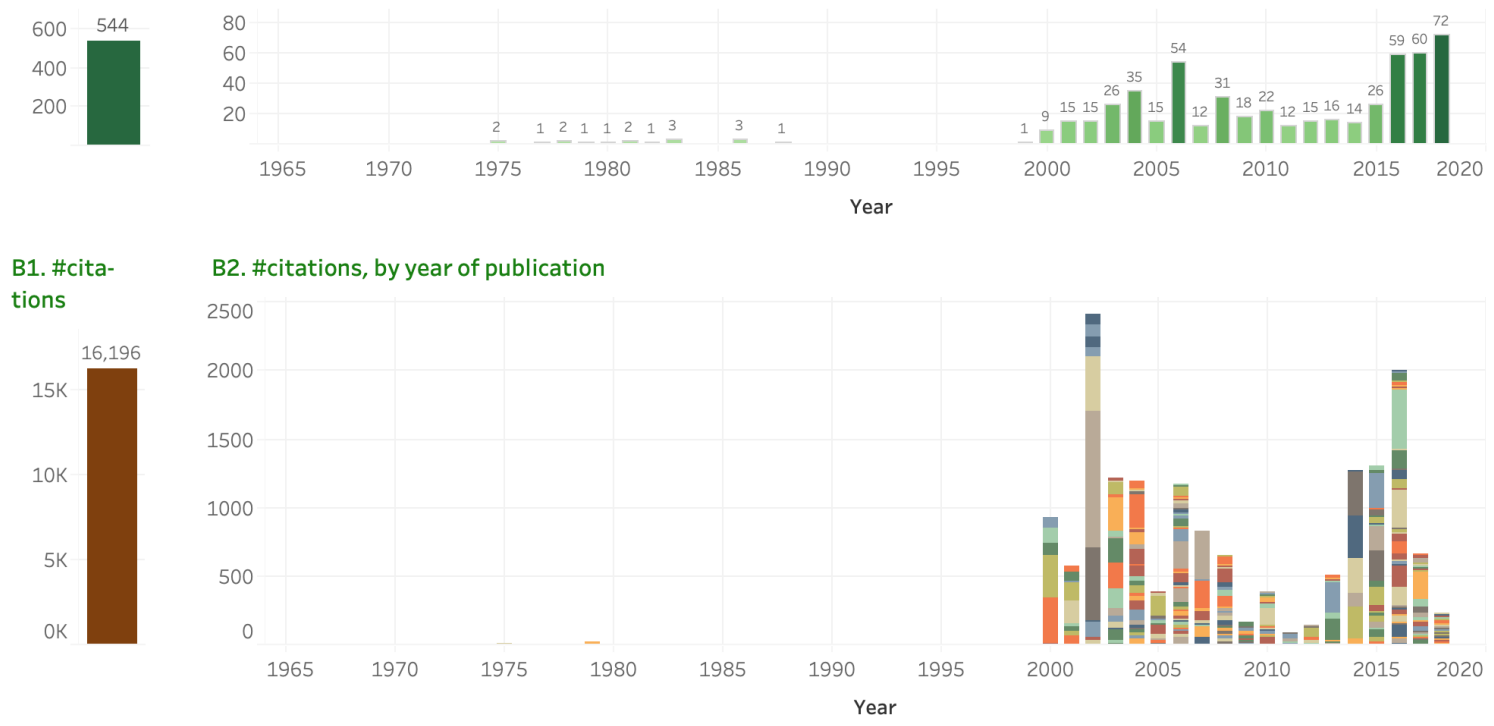

\begin{tabular}{|c|c|c|c|c|c|}
\hline Row & Paper-Id & Paper-Title & Author(s) & Year & Url \\
\hline 1 & P02-1006 & Learning surface text patterns for a Question Answering System & Ravichandran, Deepak and Hov.. & 2002 & htt.. $\quad 1,001.0$ \\
\hline 2 & D16-1044 & Multimodal Compact Bilinear Pooling for Visual Question Answering an.. & Fukui, Akira and Park, Dong Huk.. & 2016 & htt.. $\quad 430.0$ \\
\hline 3 & W02-1033 & An Analysis of the AskMSR Question-Answering System & Brill, Eric and Dumais, Susan an.. & 2002 & htt.. $\square 384.0$ \\
\hline 4 & D07-1002 & Using Semantic Roles to Improve Question Answering & Shen, Dan and Lapata, Mirella & 2007 & htt.. $\quad 354.0$ \\
\hline 5 & P02-1005 & Performance Issues and Error Analysis in an Open-Domain Question Ans.. & Moldovan, Dan and Pasca, Mari.. & 2002 & htt.. $\quad 350.0$ \\
\hline 6 & D14-1067 & Question Answering with Subgraph Embeddings & Bordes, Antoine and Chopra, Su.. & 2014 & htt.. $\square 318.0$ \\
\hline 7 & P14-1090 & Information Extraction over Structured Data: Question Answering with .. & Yao, Xuchen and Van Durme, Be.. & 2014 & $\begin{array}{lll}\text { htt.. } & 256.0 \\
\end{array}$ \\
\hline 8 & N16-1181 & Learning to Compose Neural Networks for Question Answering & Andreas, Jacob and Rohrbach, ... & 2016 & htt.. $\quad 255.0$ \\
\hline 9 & D14-1070 & A Neural Network for Factoid Question Answering over Paragraphs & Iyyer, Mohit and BoydGraber, J.. & 2014 & htt.. 255.0 \\
\hline 10 & D15-1237 & WikiQA: A Challenge Dataset for Open-Domain Question Answering & Yang, Yi and Yih, Wentau and M.. & 2015 & htt.. $\square 250.0$ \\
\hline
\end{tabular}

\section{Authors}

Row Author-name

1 Harabagiu, Sanda

2 Moldovan Dan

3 Hovy, Eduard

4 Ravichandran, Deep.

Yih, Wentau

Pasca, Marius

Meek, Christopher

Rohrbach, Marcus

Darrell, Trevor

10 Molla, Diego

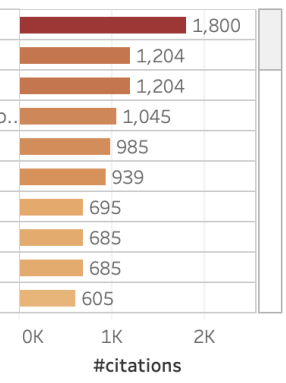

\#citations
E. Search by year of publication, title term (unigram, bigram), or author name Year of publication

$$
1965
$$

Unigram

Bigram

Author Name

\section{F3. Title Bigrams}

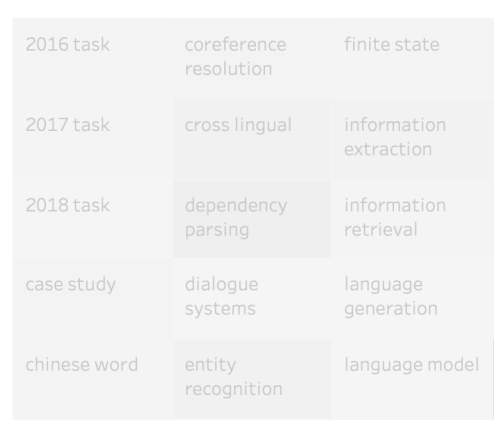

\#papers

175

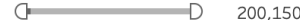

question

answering
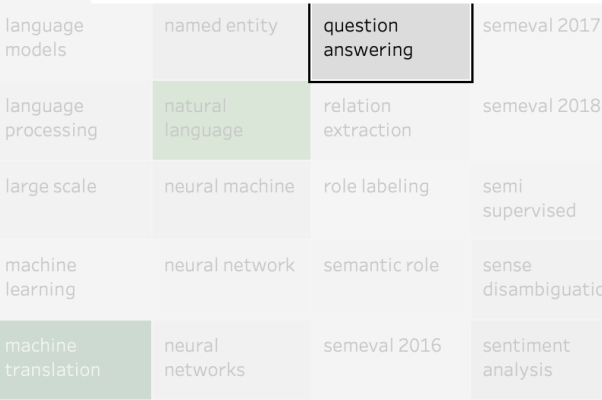

role labeling

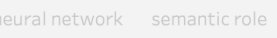

semeval 2016

Figure 17: NLP Scholar: After clicking on 'question answering' in the bigrams treemap (F3). 

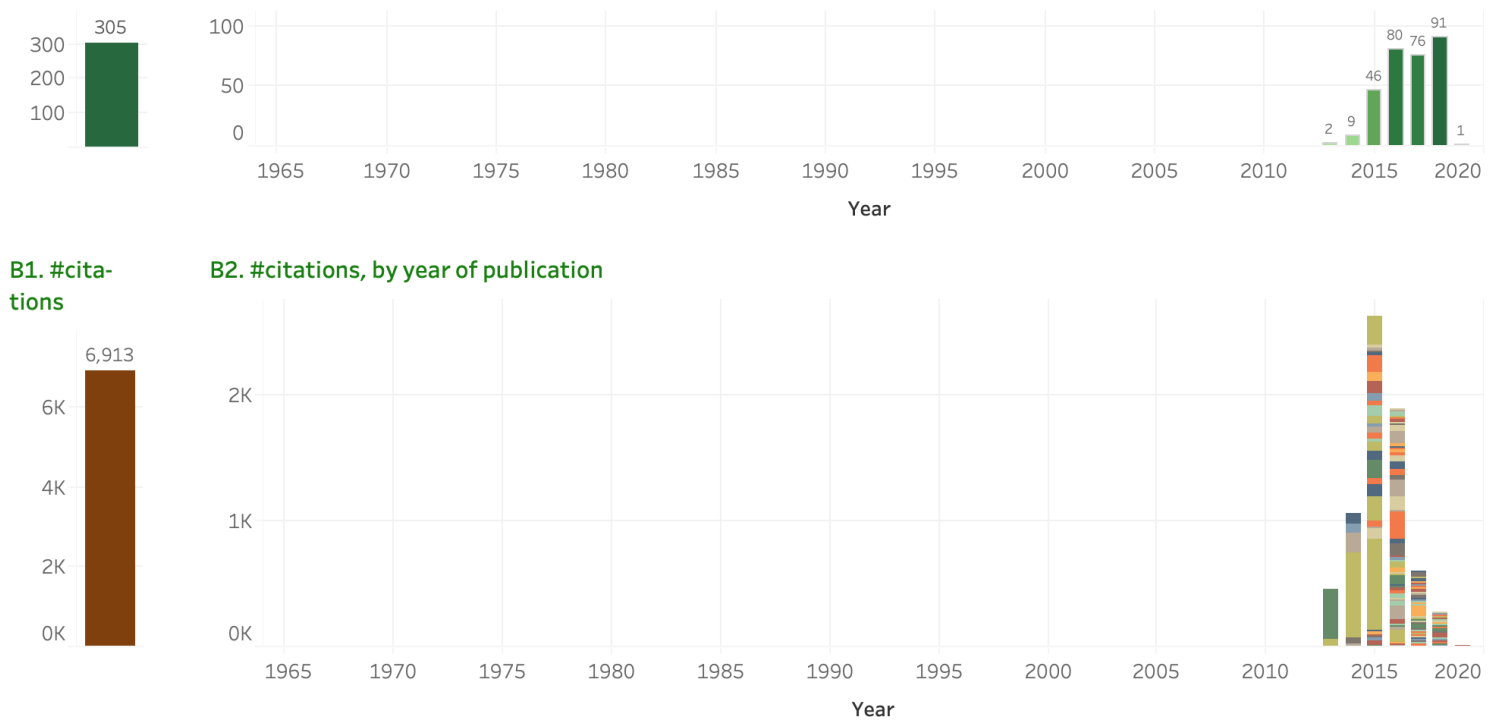

\section{Papers}

Author(s)

Year Url

Q15-1016 Improving Distributional Similarity with Lessons Learned from Word E.. Levy, Omer and Goldberg, Yoav .. 2015 htt..

D13-1141 Bilingual Word Embeddings for Phrase-Based Machine Translation $\quad$ Zou, Will Y. and Socher, Richard .. 2013 htt.

D15-1036 Evaluation methods for unsupervised word embeddings Schnabel, Tobias and Labutov L., 2015 htt.. 233.

P16-1141 Diachronic Word Embeddings Reveal Statistical Laws of Semantic Chan.. Hamilton, William L. and Leskov.. 2016 htt.. 215.0

P15-1077 Gaussian LDA for Topic Models with Word Embeddings $\quad$ Das, Rajarshi and Zaheer, Manz.. 2015 htt.. 152.0

P14-1113 Learning Semantic Hierarchies via Word Embeddings $\quad$ Fu, Ruiji and Guo, Jiang and Qin,.. 2014 htt.. 152.0

D15-1168 Fine-grained Opinion Mining with Recurrent Neural Networks and Word.. Liu, Pengfei and Joty, Shafiq an.. 2015 htt.. 130.0

P16-1035 Query Expansion with Locally-Trained Word Embeddings $\quad$ Diaz, Fernando and Mitra, Bhas.. 2016 htt.. 127.0

10 P15-2070 PPDB 2.0: Better paraphrase ranking, fine-grained entailment relations.. Pavlick, Ellie and Rastogi, Push.. 2015 htt.. 120.0

\#citations

\section{Authors}

Row Author-name

1 Goldberg, Yoav

2 Levy Omer

3 Dagan, Ido

4 Manning, Christoph

Dyer, Chris

6 Zou, Will Y.

7 Socher, Richard

Cer, Daniel

Labaka, Gorka
E. Search by year of publication, title term (unigram, bigram), or author name Year of publication

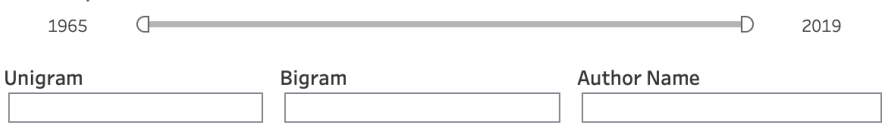

\section{F3. Title Bigrams}

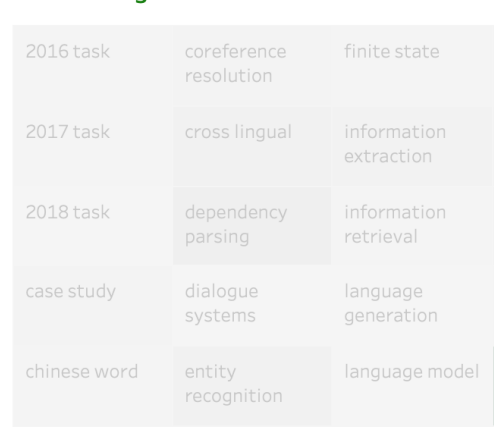

\#papers

175

200,150

Figure 18: NLP Scholar: After clicking on 'word embeddings' in the bigrams treemap (F3). 

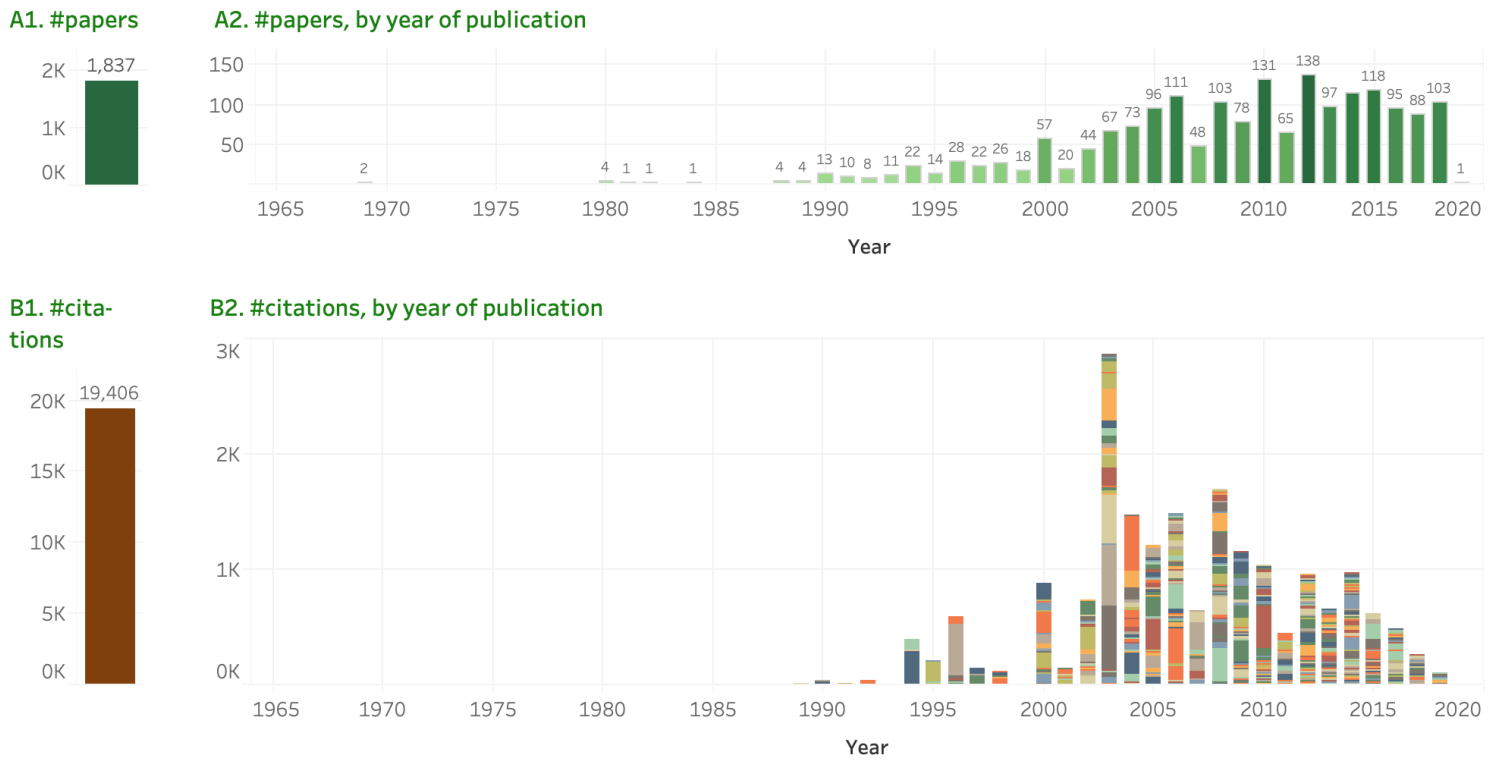

\begin{tabular}{|c|c|c|c|c|c|}
\hline Row & Paper-Id & Paper-Title & Author(s) & Year & Url \\
\hline 1 & W03-1730 & HHMM-based Chinese Lexical Analyzer ICTCLAS & Zhang, HuaPing and Yu, HongKu.. & 2003 & htt.. 545.0 \\
\hline 2 & W03-1728 & Chinese Word Segmentation as LMR Tagging & Xue, Nianwen and Shen, Libin & 2003 & 517.0 \\
\hline 3 & C04-1081 & Chinese Segmentation and New Word Detection using Conditional Rand. & Peng, Fuchun and Feng, Fangfa. & 2004 & 480.0 \\
\hline 4 & J96-3004 & A Stochastic Finite-State Word-Segmentation Algorithm for Chinese & Sproat, Richard and Shih, Chilin .. & 1996 & htt.. $\quad 450.0$ \\
\hline 5 & W03-1719 & The First International Chinese Word Segmentation Bakeoff & Sproat, Richard and Emerson, T.. & 2003 & htt.. $\quad 406.0$ \\
\hline 6 & C10-3004 & LTP: A Chinese Language Technology Platform & Che, Wanxiang and $\mathrm{Li}$, Zhenghu.. & 2010 & htt.. 367.0 \\
\hline 7 & W06-3812 & Chinese Whispers - an Efficient Graph Clustering Algorithm and its Appli.. & Biemann, Chris & 2006 & htt.. 310.0 \\
\hline 8 & P94-1012 & ALIGNING A PARALLEL ENGLISH-CHINESE CORPUS STATISTICALLY WITH... & Wu, Dekai & 1994 & htt.. 295.0 \\
\hline 9 & W08-0336 & Optimizing Chinese Word Segmentation for Machine Translation Perfor.. & Chang, PiChuan and Galley, Mic.. & 2008 & htt.. $\quad 293.0$ \\
\hline 10 & P03-1056 & Is it Harder to Parse Chinese, or the Chinese Treebank? & Levy, Roger and Manning, Chris.. & 2003 & htt.. $\quad 272.0$ \\
\hline
\end{tabular}

\section{Authors}

Row Author-name

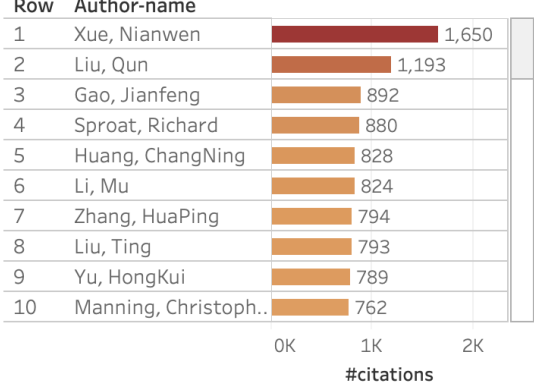

F4. Languages
E. Search by year of publication, title term (unigram, bigram), or author name Year of publication

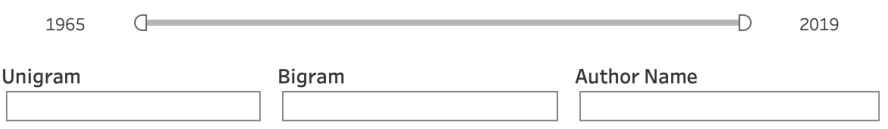

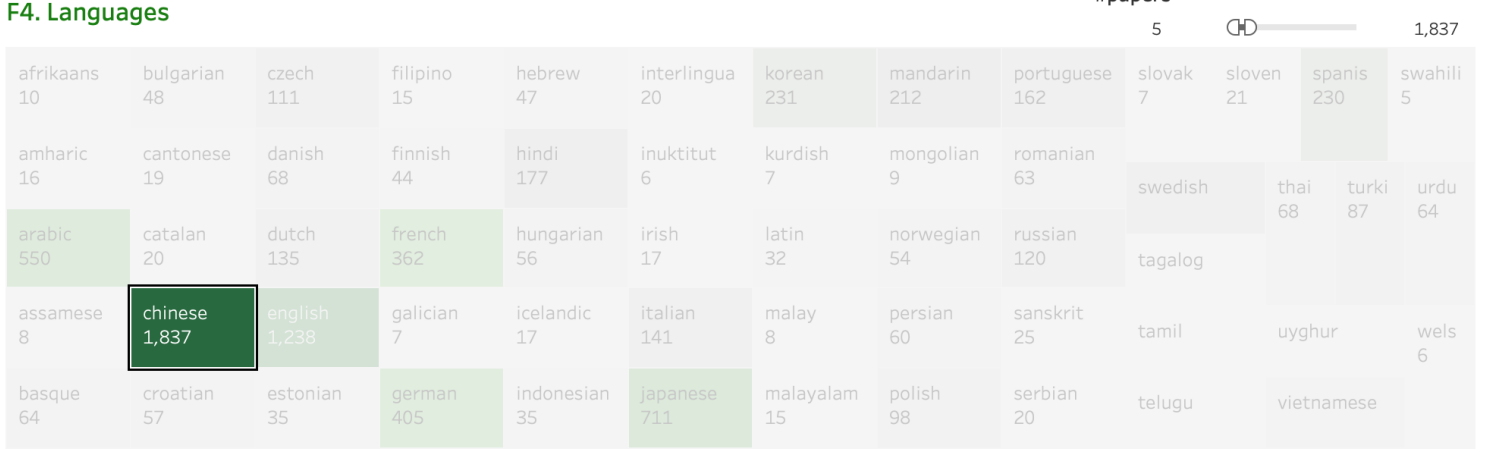

Figure 19: NLP Scholar: After clicking on 'Chinese' in the languages treemap (F4). 


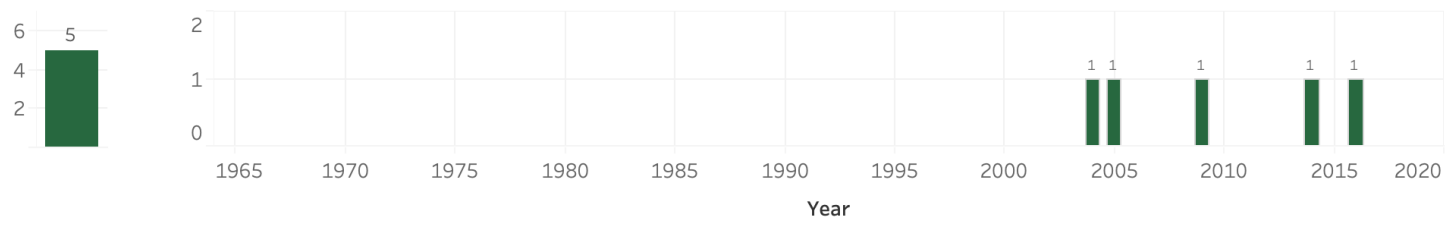

B1. \#cita- B2. \#citations, by year of publication

tions
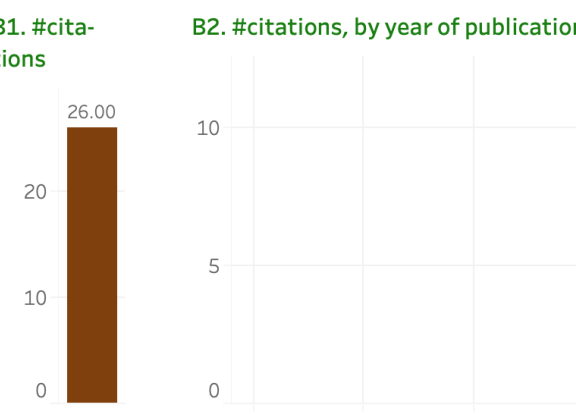

10

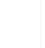

5

0

1965

1970

1975

1980

1985

1990

1995

Year

\section{Papers}

Row Paper-ld Paper-Title

Author(s)

Year Url

W05-0504 Refining the SED Heuristic for Morpheme Discovery: Another Look at S. Hu, Yu and Matveeva, Irina and .. 2005 htt. W16-5803 Word-Level Language Identification and Predicting Codeswitching Point.. Piergallini, Mario and Shirvani,.. 2016 htt. L14-1-686 Morphological parsing of Swahili using crowdsourced lexical resources Littell, Patrick and Price, Kaitly.. 2014 htt.. W09-0702 The SAWA Corpus: A Parallel Corpus English - Swahili Pauw, Guy De and Wagacha, Pe.. 2009 htt.

C04-1037 Optimizing disambiguation in Swahili $\quad$ Hurskainen, Arvi 2004 htt.

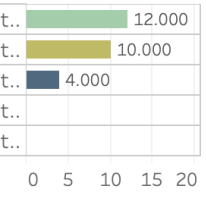

\#citations

\section{Authors}

Row Author-name

1 Sprague, Colin

2 Matveeva, Irina

$\mathrm{Hu}, \mathrm{Yu}$
Goldsmith, John

Goldsmith, John

Shirvani, Rouzbeh

Piergallini, Mario

Gautam, Gauri Shan

Price, Kaitlyn

Littell, Patrick
E. Search by year of publication, title term (unigram, bigram), or author name Year of publication

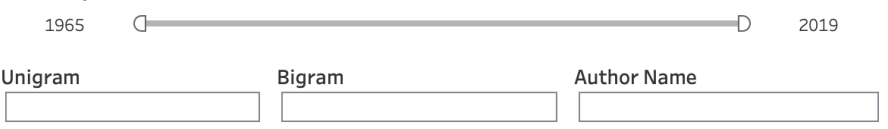

\#papers

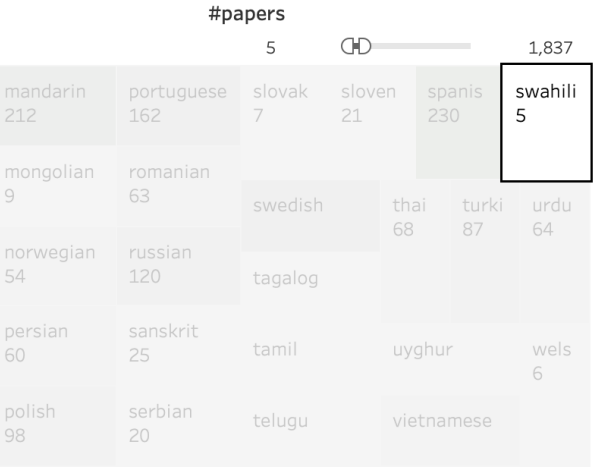

F4. Languages

Figure 20: NLP Scholar: After clicking on 'Swahili' in the languages treemap (F4). 